\title{
An Innovative Tri-rotor Drone and Associated Distributed Aerial Drone Swarm Control
}

DOI:

10.1016/j.robot.2018.02.019

\section{Document Version}

Accepted author manuscript

Link to publication record in Manchester Research Explorer

\section{Citation for published version (APA):}

Hu, J., \& Lanzon, A. (2018). An Innovative Tri-rotor Drone and Associated Distributed Aerial Drone Swarm Control. Robotics and Autonomous Systems, 103, 162-174. https://doi.org/10.1016/j.robot.2018.02.019

\section{Published in:}

Robotics and Autonomous Systems

\section{Citing this paper}

Please note that where the full-text provided on Manchester Research Explorer is the Author Accepted Manuscript or Proof version this may differ from the final Published version. If citing, it is advised that you check and use the publisher's definitive version.

\section{General rights}

Copyright and moral rights for the publications made accessible in the Research Explorer are retained by the authors and/or other copyright owners and it is a condition of accessing publications that users recognise and abide by the legal requirements associated with these rights.

\section{Takedown policy}

If you believe that this document breaches copyright please refer to the University of Manchester's Takedown Procedures [http://man.ac.uk/04Y6Bo] or contact uml.scholarlycommunications@manchester.ac.uk providing relevant details, so we can investigate your claim.

\section{OPEN ACCESS}




\title{
An Innovative Tri-rotor Drone and Associated Distributed Aerial Drone Swarm Control
}

\author{
Junyan $\mathrm{Hu}^{*}$, Alexander Lanzon \\ School of Electrical and Electronic Engineering, The University of Manchester, Manchester, M13 9PL, UK
}

\begin{abstract}
This paper presents a novel unmanned aerial vehicle platform based on a three rotor configuration, which can achieve the highest level of manoeuvrability in all 6 dimensions (i.e. 3D position and 3D attitude). The three propellers can be tilted independently to obtain full force and torque vectoring authority, such that this new aerial robotic platform can overcome the limitations of a classic quadrotor UAV that can not change its attitude while hovering at a stationary position. A robust feedback linearization controller is developed to deal with this highly coupled and nonlinear dynamics of the proposed tri-rotor UAV, which linearises the dynamics globally using geometric transformations to produce a linear model that matches the Jacobi linearization of the nonlinear dynamics at the operating point of interest. A distributed formation control tracking protocol is then proposed to control a swarm of tri-rotor UAVs. The 3D position and 3D attitude of each vehicle can be controlled independently to follow a desired time-varying formation. The effectiveness of the designed control strategy is illustrated in a realistic virtual reality simulation environment based on real hardware parameters from a physical construction.
\end{abstract}

Keywords:

Aerial robotics, distributed systems, formation control, optimal control, multi-agent, unmanned aerial vehicles.

\section{Introduction}

In recent years, cooperative control of multi-rotor Unmanned Aerial Vehicles (UAVs) have received significant attention from both the practical engineering and academic communities due to their broad prospect in applications [1]. When working together, they are able to perform complex tasks with excellent efficiency and reliability, such as search and rescue [2], crop and weed management in agriculture [3], oil pipeline surveillance [4], etc. Aiming at more efficient configurations in terms of size, autonomy, flight range, payload capacity and other factors, some innovative vehicle platforms are developed by researchers [5]. One of such aerial robotic platforms that holds new and significant properties is the tri-rotor UAV, which is cost effective with more flexibility and agility [6],[7].

The proposed tri-rotor UAV has three rotors arranged in an equilateral triangular configuation and each rotor is attached to a servo motor that can independently change the rotating direction of the propeller. Thus, complete 3D thrust and 3D torque vectoring authority is achieved, which means that the vehicle does not have a nominally upright flying orientation: it can fly in any orientation chosen by the user. Any time-dependent 3D position trajectory can be tracked at the same time as tracking any time-dependent 3D attitude trajectory. This configuration guarantees the UAV a high level of flexibility and maneuverability for attitude control and position movement. Compared to the quadrotor, this innovative configuration also requires less

\footnotetext{
${ }^{*}$ Corresponding author

Email addresses: Junyan.Hu@manchester . ac .uk (Junyan Hu), Alexander. Lanzon@manchester. ac.uk (Alexander Lanzon)
}

hover power and hence provides longer flight time [8], which makes it ideal for deployment in various missions.

To the best of the authors' knowledge, no prior literature has studied a tri-rotor UAV configuration with completely independent tilted-rotor capability on all three rotors. The tri-rotor UAV introduced in [9] only has one servo motor that is installed on the arm, which can not hold different attitudes while hovering. A triangular quadrotor is proposed in [8], which contains a single large rotor fixed on the main body. This configuration requires more power to hover and causes uncompensated gyroscopic drift.

In contrast to a quadrotor UAV, which has zero angular momentum in hover, a tri-rotor UAV has persistent angular moment, and hence also gyroscopic dynamics due to the asymmetric configuration of the system which poses significant control systems complexities. Furthermore, independent attitude and trajectory tracking can and should be considered simultaneously. However, the control algorithm in [10] only considers attitude stabilization (as opposed to simultaneous independent attitude and trajectory tracking) and the control design proposed in [11] only focus on the static hovering. In this paper, both these two objectives (i.e. simultaneous independent 3D attitude and 3D trajectory tracking) are considered for the tri-rotor UAV in order to overcome the limitation of quadrotors and thus create more possibilities when performing special tasks through aerial robotic platforms.

Furthermore, swarm robotics is a field of multi-robotics where a group of robots are controlled in a distributed way to perform complex tasks in a more efficient way than using a single robot [12]. As a key control technique in swarm 
robotics, distributed cooperative control of multi-agent systems has also experienced a rapid growth in the research efforts from the international robotics community, which includes consensus control [13],[14], rendezvous control [15], obstacle avoidance [16], formation control [17],[18], etc. Formation control of multi-agent systems is hence a key active area of research which shows broad applications [19]. In applications where the goal cannot be accomplished by a single robot or a single aerial robotic vehicle due to physical limitations in its capability, formation control has been flagged as an important underpinning methodology. It can be applied to a variety of areas, such as cooperative surveillance [20], target enclosing [21], load transport [22], etc. Based on a consensus strategy, [23] proved that leader-follower, virtual structure and behavior-based formation control approaches can be unified in the framework of consensus problems. [24] discussed the formation stability problems for general high-order swarm systems, but the question how to achieve desired formation was not considered. Static formation experiments on quadrotor swarm systems based on consensus approaches is achieved in [25], while time-varying formation control of aerial swarm systems is still a vigorously active research topic with much progress still needed.

Motivated by the challenges stated above, the combination of time-varying formation control and the proposed innovative tri-rotor drone is developed and investigated in this paper. The formation control protocol for the designed aerial swarm is fully distributed. The communication topology of the network is modelled using graph theory. Robust feedback linearization [26] is used to handle the tri-rotor drone's highly coupled and nonlinear dynamics. It provides a systematic multi-input/multioutput (MIMO) method which linearises nonlinear dynamics geometrically to match the Jacobi linearization of the nonlinear system at the operating point of interest. In contrast to classic feedback linearization which does full nonlinear dynamic inversion to produce a linear system which is simply a chain of integrators, robust feedback linearization preserves the system information at the operating point of interest. It has been successfully demonstrated [27] to provide significant robustness to both model uncertainty and external dynamics. An output feedback formation control protocol is also applied to the networked tri-rotor UAV swarm, which consists of an optimal state observer and an optimal (Linear Quadratic Regulator-LQR) distributed state feedback formation protocol. It is shown that LQR based optimal design provides a straightforward way to construct fully distributed controllers and observers that ensure stabilization and synchronization of the swarm [28].

The paper is organized as follows. Notation and preliminaries on algebraic graph theory are presented in Section 2 . The nonlinear dynamical model of the tri-rotor drone is described in Section 3. Robust feedback linearization of a single tri-rotor drone is first given in Section 4 and then an optimal distributed formation controller is designed at the end of Section 4 to control a swarm of tri-rotor drones. Section 5 is devoted to the presentation of simulation results when the proposed control architecture is applied to the aerial swarm of tri-rotor drones. Conclusions are given in Section 6.

\section{Preliminaries}

In this section, notation, definitions and basic concepts on graph theory are introduced.

\subsection{Notation and Definitions}

Let $\boldsymbol{I}_{n} \in \mathbb{R}^{n \times n}$ denote the identity matrix of demension $n$ and $\mathbf{1}_{N} \in \mathbb{R}^{n}$ be the vector with all entries equal to one. $\operatorname{diag}\left\{a_{i}\right\}$ represents a diagonal matrix with diagonal entries $a_{i}$. The Kronecker product is denoted by $\otimes$. We use the superscript $T$ and * to denote the transpose and complex conjugate transpose of a matrix respectively. For $\lambda \in \mathbb{C}, \operatorname{Re}(\lambda)$ is the real part of $\lambda$.

\subsection{Graph Theory}

Consider a weighted and directed graph $\mathcal{G}=(\mathcal{V}, \mathcal{E}, \mathcal{A})$ with a nonempty set of $N$ nodes $\mathcal{V}=\{1,2, \ldots, N\}$, a set of edges $\mathcal{E} \subset \mathcal{V} \times \mathcal{V}$, and associated adjacency matrix $\mathcal{A}=\left[a_{i j}\right] \in \mathbb{R}^{N \times N}$. An edge rooted at node $i$ and ended at node $j$ is denoted by $(i, j)$, which means information can flow from node $i$ to node $j . a_{i j}$ is the weight of edge $(i, j)$ and $a_{i j}>0$ if $(i, j) \in \mathcal{E}$. Assume that there are no repeated edges and no self loops. Node $j$ is called a neighbour of node $i$ if $(i, j) \in \mathcal{E}$. Define the in-degree matrix as $\boldsymbol{D}=\operatorname{diag}\left\{d_{i}\right\} \in \mathbb{R}^{N \times N}$ with $d_{i}=\sum_{j=1}^{N} a_{i j}$. The Laplacian matrix $\boldsymbol{L} \in \mathbb{R}^{N \times N}$ of $\mathcal{G}$ is defined as $\boldsymbol{L}=\boldsymbol{D}-\mathcal{A}$. A directed graph has or contains a directed spanning tree if there exists a node, called the root, such that there exists a directed path from this node to every other nodes.

Lemma 1 ([29]). If $\mathcal{G}$ contains a spanning tree, then zero is a simple eigenvalue of $\boldsymbol{L}$ with associated right eigenvector $\mathbf{1}_{N}$, and all the other $N-1$ eigenvalues have nonnegative real parts.

The following assumption of graph topology holds throughout this paper.

Assumption 1. The directed graph $\mathcal{G}$ contains a spanning tree and the root node $i$ can obtain information from the leader node.

\section{Mathematical Modeling}

In this section, we dynamically modeling the proposed trirotor UAV.

\subsection{System Description}

The configuration of the tri-rotor UAV is illustrated in Fig. 1, which was first proposed in our earlier work [6]. The UAV has a triangular structure with three arms and a force generating unit plus a revolute joint at the end of each arm. All three arms have identical length $l$. Each force generating unit includes a fixed pitch propeller driven by a brushless DC motor to provide thrust. The motors can be powered by a single battery pack located at the centre of mass or by three separate battery packs located at an equal distance from the centre of mass and each other. The propeller-motor assembly is attached to the body arm via a servo motor that can rotate in a vertical plane to tilt the propeller-motor assembly with an angle $\alpha_{s i}$ (the subscript 


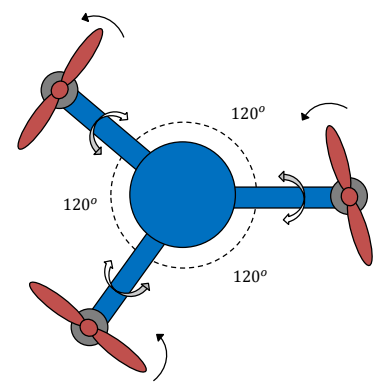

(a)

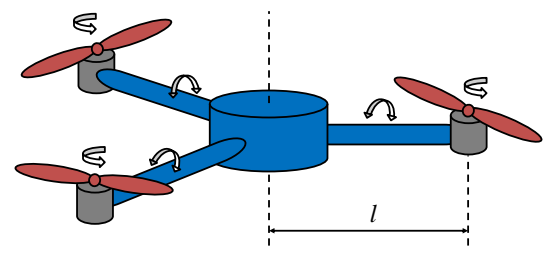

(b)

Fig. 1. Design of the tri-rotor UAV. (a) Top view. (b) 3D view

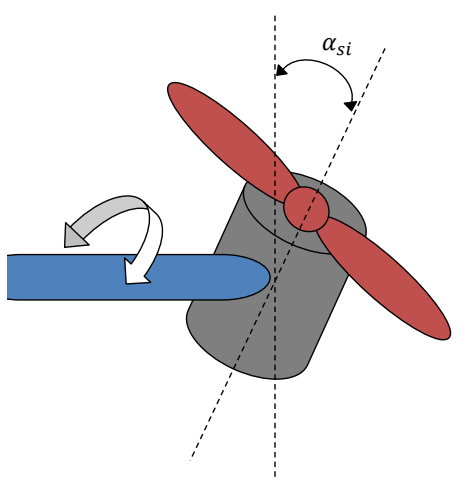

Fig. 2. Front view of one arm

's' denotes servo) in order to produce a horizontal component of the generated force, which is shown in Fig. 2.

All three propellers can be tilted independently to give full thrust vectoring authority. Then the UAV becomes a full sixdegrees-of-freedom (6-DOF) vehicle in which all motions can be achieved independently by changing speed of the propellers and tilting angles of the servo motor directly. This configuration enables vehicle attitude (i.e. 3D orientation) and vehicle translation (i.e. 3D movement) to be independently controlled.

In order to develop the dynamic model of the proposed trirotor UAV, the following right hand coordinate systems shown in Fig. 3 are considered: $\left(X_{e}, Y_{e}, Z_{e}\right)$ represents the earth coordinate system, which is assumed to be inertial (i.e. fixed). $\left(X_{b}, Y_{b}, Z_{b}\right)$ denotes the body coordinate system, where the origin $O_{b}$ is fixed to the center of mass of the vehicle. This coordinate system moves with the vehicle. $\left(X_{l i}, Y_{l i}, Z_{l i}\right)$ with $i \in\{1,2,3\}$ is the local coordinate system of each propellermotor assembly. The location of the origin of each local coordinate system coincides with the intersection of the UAV arm and the propeller-motor assembly, where $X_{l i}$ is extended out-

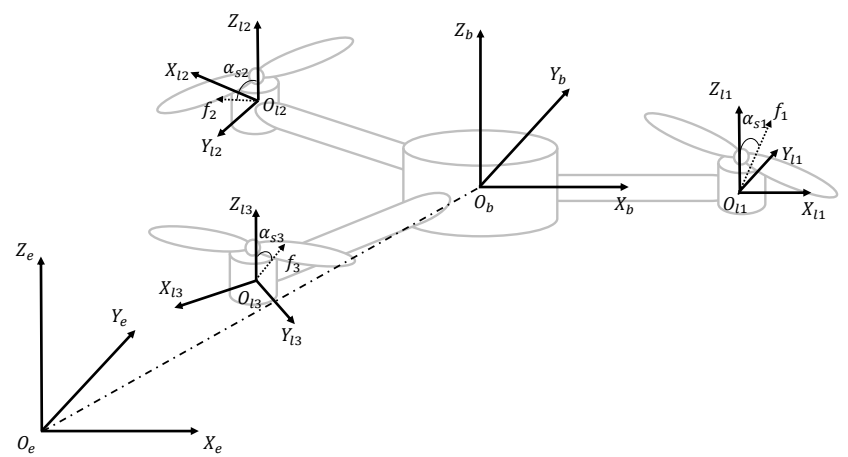

Fig. 3. Coordinate systems of the tri-rotor UAV

side the $i^{\text {th }}$ arm of UAV along the same line as the arm and $Z_{l i}$ is along the direction of the motor shaft axis when the servo angle is zero.

In this section, the superscript $b, e$ and $l_{i}$ are used to denote the corresponding coordinate system in which vectors are expressed. The subscript $i$ refers to the $i^{\text {th }}$ propeller, servo motor or brushless DC motor with $i \in\{1,2,3\}$. The nominal mathematical model is based on the following assumptions:

1) Fast actuators are assumed, so the dynamics of actuators are neglected.

2) Propellers are considered to be rigid, thus blade flapping is not considered in the model.

3) The body structure is rigid and the mass is fixed.

It should be noted that although we do not consider these factors in model design, they can still be included as perturbations and uncertainties when carrying out simulation or experiment to test the robustness of the proposed control system in the next section.

In order to obtain the dynamical equations of motion of the vehicle, both forces and torques acting on the UAV need to be analyzed.

\subsection{Forces Analysis}

There are two main forces acting on the tri-rotor, which are the propulsive force and the gravitational force respectively.

The total propulsive force $\boldsymbol{F}_{p}^{b}$ is equal to the algebraic sum of the three individual propulsive forces produced by each propeller. The individual propulsive forces $\boldsymbol{F}_{p_{i}}^{l_{i}}$ at the local coordinate systems are given by:

$$
\boldsymbol{F}_{p_{i}}^{l_{i}}=\left[\begin{array}{c}
0 \\
k_{f} \omega_{m i}^{2} \sin \left(\alpha_{s_{i}}\right) \\
k_{f} \omega_{m i}^{2} \cos \left(\alpha_{s_{i}}\right)
\end{array}\right], \quad i \in\{1,2,3\},
$$

where $k_{f}$ is the thrust coefficient of the propeller that can be easily determined from static thrust tests [30], $\omega_{m i}$ is the rotational speed of the $i^{\text {th }}$ brushless DC motor and $\alpha_{s i}$ is the tilting angle of the $i^{\text {th }}$ servo motor.

To obtain the propulsive forces in the body coordinate system, consider the following rotation matrices from propeller 
local coordinate systems $l_{1}, l_{2}$ and $l_{3}$ to the body coordinate system $b$ as

$$
\begin{aligned}
\boldsymbol{R}_{l_{1}}^{b} & =\left[\begin{array}{lll}
1 & 0 & 0 \\
0 & 1 & 0 \\
0 & 0 & 1
\end{array}\right], \boldsymbol{R}_{l_{2}}^{b}=\left[\begin{array}{ccc}
-\frac{1}{2} & -\frac{\sqrt{3}}{2} & 0 \\
\frac{\sqrt{3}}{2} & -\frac{1}{2} & 0 \\
0 & 0 & 1
\end{array}\right], \\
\boldsymbol{R}_{l_{3}}^{b} & =\left[\begin{array}{ccc}
-\frac{1}{2} & \frac{\sqrt{3}}{2} & 0 \\
-\frac{\sqrt{3}}{2} & -\frac{1}{2} & 0 \\
0 & 0 & 1
\end{array}\right] .
\end{aligned}
$$

The total propulsive force is then given by

$$
\boldsymbol{F}_{p}^{b}=\boldsymbol{R}_{l_{1}}^{b} \boldsymbol{F}_{p_{1}}^{l_{1}}+\boldsymbol{R}_{l_{2}}^{b} \boldsymbol{F}_{p_{2}}^{l_{2}}+\boldsymbol{R}_{l_{3}}^{b} \boldsymbol{F}_{p_{3}}^{l_{3}}=k_{f} \boldsymbol{H}_{f} \boldsymbol{\rho}
$$

where

$$
\boldsymbol{H}_{f}=\left[\begin{array}{cccccc}
0 & -\frac{\sqrt{3}}{2} & \frac{\sqrt{3}}{2} & 0 & 0 & 0 \\
1 & -\frac{1}{2} & -\frac{1}{2} & 0 & 0 & 0 \\
0 & 0 & 0 & 1 & 1 & 1
\end{array}\right] \text { and } \rho=\left[\begin{array}{c}
\omega_{m 1}^{2} \sin \left(\alpha_{s_{1}}\right) \\
\omega_{m 2}^{2} \sin \left(\alpha_{s_{2}}\right) \\
\omega_{m 3}^{2} \sin \left(\alpha_{s_{3}}\right) \\
\omega_{m 1}^{2} \cos \left(\alpha_{s_{1}}\right) \\
\omega_{m 2}^{2} \cos \left(\alpha_{s_{2}}\right) \\
\omega_{m 3}^{2} \cos \left(\alpha_{s_{3}}\right)
\end{array}\right] \text {. }
$$

The gravitational force in the earth coordinate system can be written as

$$
\boldsymbol{F}_{g}^{e}=\left[\begin{array}{c}
0 \\
0 \\
-m g
\end{array}\right]
$$

where $g$ is the gravity acceleration and $m$ is the total mass of the tri-rotor UAV.

In the body coordinate system, we have

$$
\boldsymbol{F}_{g}^{b}=\boldsymbol{R}_{e}^{b} \boldsymbol{F}_{g}^{e}
$$

where $\boldsymbol{R}_{e}^{b}$ is the rotation matrix from frame $e$ to frame $b$.

The notation of attitude angles for the UAV is roll angle $\phi$, pitch angle $\theta$ and yaw angle $\psi$, which represents counterclockwise rotation of angles about the $X_{b}$-axis, $Y_{b}$-axis and $Z_{b}$-axis respectively. The rotation matrices are given by

$$
\begin{aligned}
& \boldsymbol{R}_{x}(\phi)=\left[\begin{array}{ccc}
1 & 0 & 0 \\
0 & \cos (\phi) & -\sin (\phi) \\
0 & \sin (\phi) & \cos (\phi)
\end{array}\right], \\
& \boldsymbol{R}_{y}(\theta)=\left[\begin{array}{ccc}
\cos (\theta) & 0 & \sin (\theta) \\
0 & 1 & 0 \\
-\sin (\theta) & 0 & \cos (\theta)
\end{array}\right], \\
& \boldsymbol{R}_{z}(\psi)=\left[\begin{array}{ccc}
\cos (\psi) & -\sin (\psi) & 0 \\
-\sin (\psi) & \cos (\psi) & 0 \\
0 & 0 & 1
\end{array}\right] .
\end{aligned}
$$

According to the properties of the rotation matrix we have $\boldsymbol{R}_{e}^{b}=\left(\boldsymbol{R}_{b}^{e}\right)^{-1}$, where $\boldsymbol{R}_{b}^{e}$ is the rotation matrix from body coordinate system to the earth coordinate system given by

$$
\begin{aligned}
\boldsymbol{R}_{b}^{e} & =\boldsymbol{R}_{z} \boldsymbol{R}_{y} \boldsymbol{R}_{x} \\
& =\left[\begin{array}{ccc}
C_{\theta} C_{\psi} & C_{\psi} S_{\theta} S_{\phi}-C_{\phi} S_{\psi} & C_{\phi} C_{\psi} S_{\theta}+S_{\phi} S_{\psi} \\
C_{\theta} S_{\psi} & S_{\theta} S_{\phi} S_{\psi}+C_{\phi} C_{\psi} & C_{\phi} S_{\theta} S_{\psi}-C_{\psi} S_{\phi} \\
-S_{\theta} & C_{\theta} S_{\phi} & C_{\phi} C_{\theta}
\end{array}\right],
\end{aligned}
$$

where $S_{(.)}$and $C_{(.)}$represent $\sin ($.$) and \cos ($.$) respectively.$

Then the gravitational force in the body coordinate system can be expressed by

$$
\boldsymbol{F}_{g}^{b}=m g \boldsymbol{\Theta},
$$

where

$$
\boldsymbol{\Theta}=\left[\begin{array}{c}
\sin (\theta) \\
-\sin (\phi) \cos (\theta) \\
-\cos (\phi) \cos (\theta)
\end{array}\right] .
$$

Thus, the total force acting on the tri-rotor in the body coordinate system can be written as

$$
\boldsymbol{F}^{b}=\boldsymbol{F}_{p}^{b}+\boldsymbol{F}_{g}^{b}=k_{f} \boldsymbol{H}_{f} \boldsymbol{\rho}+m g \boldsymbol{\Theta} .
$$

\subsection{Torques Analysis}

There are two main torques acting on the tri-rotor, which are the propulsive torque and drag torque.

The propulsive torque is the torque caused by the thrust generated from the propellers near the centre of mass of the UAV. Since there are three identical arms with length $l$, the propulsive torque for each actuator is given by

$$
\boldsymbol{\tau}_{p i}^{b}=\boldsymbol{l}_{i}^{b} \times \boldsymbol{F}_{p_{i}}^{b}, \quad i \in\{1,2,3\}
$$

where

$$
\boldsymbol{l}_{1}^{b}=l\left[\begin{array}{l}
1 \\
0 \\
0
\end{array}\right], \boldsymbol{l}_{2}^{b}=l\left[\begin{array}{c}
\frac{\sqrt{3}}{2} \\
-\frac{1}{2} \\
0
\end{array}\right], \boldsymbol{l}_{3}^{b}=l\left[\begin{array}{c}
-\frac{1}{2} \\
-\frac{\sqrt{3}}{2} \\
0
\end{array}\right]
$$

and $\boldsymbol{F}_{p_{i}}^{b}=\boldsymbol{R}_{l_{i}}^{b} \boldsymbol{F}_{p_{i}}^{l_{i}}$.

Then the total propulsive torque with respect to the body coordinate system can be written as:

$$
\tau_{p}^{b}=\boldsymbol{\tau}_{p 1}^{b}+\boldsymbol{\tau}_{p 2}^{b}+\boldsymbol{\tau}_{p 3}^{b}=k_{f} \boldsymbol{H}_{t} \boldsymbol{\rho}
$$

where

$$
\boldsymbol{H}_{t}=l\left[\begin{array}{cccccc}
0 & 0 & 0 & 0 & \frac{\sqrt{3}}{2} & -\frac{\sqrt{3}}{2} \\
0 & 0 & 0 & -1 & \frac{1}{2} & \frac{1}{2} \\
1 & 1 & 1 & 0 & 0 & 0
\end{array}\right]
$$

The drag torque is expressed as the torque caused by the aerodynamic drag forces, which is in the opposite direction to the rotation direction of propeller. Thus, the resulting drag torque on the $i^{t h}$ propeller is given by $k_{d} \omega_{m i}^{2}$, where $k_{d}$ is the drag torque to speed coefficient resulting from the rotation of the propeller.

The drag torque on the propellers causes an equal reaction torque on the vehicle which can be expressed in the local coordinate systems as

$$
\boldsymbol{\tau}_{d_{i}}^{l_{i}}=\left[\begin{array}{c}
0 \\
-k_{d} \omega_{m i}^{2} \sin \left(\alpha_{s_{i}}\right) \\
-k_{d} \omega_{m i}^{2} \cos \left(\alpha_{s_{i}}\right)
\end{array}\right], \quad i \in\{1,2,3\} .
$$

The total drag (or reaction) torque in the body coordinate system can be written as

$$
\begin{aligned}
\boldsymbol{\tau}_{d}^{b} & =\boldsymbol{\tau}_{d_{1}}^{b}+\boldsymbol{\tau}_{d_{2}}^{b}+\boldsymbol{\tau}_{d_{3}}^{b}=\boldsymbol{R}_{l_{1}}^{b} \boldsymbol{\tau}_{d_{1}}^{l_{1}}+\boldsymbol{R}_{l_{2}}^{b} \boldsymbol{\tau}_{d_{2}}^{l_{2}}+\boldsymbol{R}_{l_{3}}^{b} \boldsymbol{\tau}_{d_{3}}^{l_{3}} \\
& =-k_{d} \boldsymbol{H}_{f} \boldsymbol{\rho} .
\end{aligned}
$$


Finally, the total torque acting on the tri-rotor UAV in the body coordinate system can be written as

$$
\boldsymbol{\tau}^{b}=\boldsymbol{\tau}_{p}^{b}+\boldsymbol{\tau}_{d}^{b}=\left(k_{f} \boldsymbol{H}_{t}-k_{d} \boldsymbol{H}_{f}\right) \boldsymbol{\rho} .
$$

\subsection{Dynamic Model}

Under the assumption stated earlier that the tri-rotor UAV is a rigid body of fixed mass, the vehicle's translational and rotational dynamics can be calculated by the Newton-Euler's second law of motion [31] in the body coordinate frame as

$$
\begin{gathered}
\boldsymbol{F}^{b}=m\left(\dot{\boldsymbol{v}}_{v}^{b}+\boldsymbol{S}\left(\boldsymbol{\omega}_{v}^{b}\right) \boldsymbol{v}_{v}^{b}\right), \\
\boldsymbol{\tau}^{b}=\boldsymbol{I}_{v}^{b} \dot{\boldsymbol{\omega}}_{v}^{b}+\boldsymbol{S}\left(\boldsymbol{\omega}_{v}^{b}\right) \boldsymbol{I}_{v}^{b} \boldsymbol{\omega}_{v}^{b},
\end{gathered}
$$

where $\boldsymbol{v}_{v}^{b}$ is the vehicle's translational velocity measured in the body coordinate frame and $\omega_{v}^{b}$ is the vehicle's angular velocity. The vehicle's inertia matrix expressed in the body coordinate frame and the skew matrix constructed from the vector $\omega_{v}^{b}=$ $\left[\begin{array}{lll}p & q & r\end{array}\right]^{T}$ are given by

$$
\boldsymbol{I}_{v}^{b}=\left[\begin{array}{ccc}
I_{x x} & 0 & 0 \\
0 & I_{y y} & -I_{y z} \\
0 & -I_{y z} & I_{z z}
\end{array}\right]
$$

and

$$
\boldsymbol{S}\left(\boldsymbol{\omega}_{v}^{b}\right)=\left[\begin{array}{ccc}
0 & -r & q \\
r & 0 & -p \\
-q & p & 0
\end{array}\right]
$$

Now, substituting $\boldsymbol{F}^{b}$ and $\boldsymbol{\tau}^{b}$ from (9) and (14) gives

$$
\begin{gathered}
k_{f} \boldsymbol{H}_{f} \boldsymbol{\rho}+m g \boldsymbol{\Theta}=m\left(\dot{\boldsymbol{v}}_{v}^{b}+\boldsymbol{S}\left(\boldsymbol{\omega}_{v}^{b}\right) \boldsymbol{v}_{v}^{b}\right), \\
\left(k_{f} \boldsymbol{H}_{t}-k_{d} \boldsymbol{H}_{f}\right) \boldsymbol{\rho}=\boldsymbol{I}_{v}^{b} \dot{\boldsymbol{\omega}}_{v}^{b}+\boldsymbol{S}\left(\boldsymbol{\omega}_{v}^{b}\right) \boldsymbol{I}_{v}^{b} \boldsymbol{\omega}_{v}^{b} .
\end{gathered}
$$

Let $\boldsymbol{\eta}_{v}=\left[\begin{array}{ll}\phi & \psi\end{array}\right]^{T}$ and $\lambda_{v}^{e}=\left[\begin{array}{lll}x_{v} & y_{v} & z_{v}\end{array}\right]^{T}$ denote respectively the attitude vector and the position vector with respect to the earth coordinate system. Then

$$
\begin{aligned}
& \dot{\boldsymbol{\eta}}_{v}=\boldsymbol{\Psi} \omega_{v}^{b}, \\
& \dot{\lambda}_{v}^{e}=\boldsymbol{R}_{b}^{e} \boldsymbol{v}_{v}^{b},
\end{aligned}
$$

describe the relations between velocities and positions [32], where $\boldsymbol{\Psi}$ relates the instantaneous angular velocities around the $X_{b}$-axis, $Y_{b}$-axis and $Z_{b}$-axis to the rate of change of the roll, pitch and yaw angles. It is given in [33] as

$$
\boldsymbol{\Psi}=\left[\begin{array}{ccc}
1 & \sin (\phi) \tan (\theta) & \cos (\theta) \tan (\theta) \\
0 & \cos (\theta) & -\sin (\phi) \\
0 & \sin (\phi) \sec (\theta) & \cos (\phi) \sec (\theta)
\end{array}\right],-\frac{\pi}{2}<\theta<\frac{\pi}{2} .
$$

Therefore, the dynamic model of the tri-rotor can be described in a compact form as

$$
\dot{\boldsymbol{v}}_{v}^{b}=g \boldsymbol{\Theta}-\boldsymbol{S}\left(\boldsymbol{\omega}_{v}^{b}\right) \boldsymbol{v}_{v}^{b}+\frac{k_{f}}{m} \boldsymbol{H}_{f} \boldsymbol{\rho},
$$

$$
\dot{\boldsymbol{\omega}}_{v}^{b}=-\left(\boldsymbol{I}_{v}^{b}\right)^{-1} \boldsymbol{S}\left(\boldsymbol{\omega}_{v}^{b}\right) \boldsymbol{I}_{v}^{b} \boldsymbol{\omega}_{v}^{b}+\left(\boldsymbol{I}_{v}^{b}\right)^{-1}\left(k_{f} \boldsymbol{H}_{t}-k_{d} \boldsymbol{H}_{f}\right) \boldsymbol{\rho}
$$

$$
\begin{aligned}
& \dot{\boldsymbol{\eta}}_{v}=\boldsymbol{\Psi} \omega_{v}^{b}, \\
& \dot{\lambda}_{v}^{e}=\boldsymbol{R}_{b}^{e} \boldsymbol{v}_{v}^{b},
\end{aligned}
$$

where

$$
\boldsymbol{v}_{v}^{b}=\left[\begin{array}{c}
u_{b} \\
v_{b} \\
w_{b}
\end{array}\right], \omega_{v}^{b}=\left[\begin{array}{c}
p \\
q \\
r
\end{array}\right], \boldsymbol{\eta}_{v}=\left[\begin{array}{c}
\phi \\
\theta \\
\psi
\end{array}\right] \text { and } \lambda_{v}^{e}=\left[\begin{array}{c}
x_{v} \\
y_{v} \\
z_{v}
\end{array}\right] .
$$

Choosing the state vector as

$$
\begin{aligned}
\boldsymbol{x} & =\left[\begin{array}{llllllllllll}
x_{1} & x_{2} & x_{3} & x_{4} & x_{5} & x_{6} & x_{7} & x_{8} & x_{9} & x_{10} & x_{11} & x_{12}
\end{array}\right]^{T} \\
& =\left[\begin{array}{llllllllllll}
u_{b} & v_{b} & w_{b} & p & q & r & \phi & \theta & \psi & x_{v} & y_{v} & z_{v}
\end{array}\right]^{T},
\end{aligned}
$$

and the input vector as

$$
\boldsymbol{u}=\boldsymbol{\rho}=\left[\begin{array}{l}
u_{1} \\
u_{2} \\
u_{3} \\
u_{4} \\
u_{5} \\
u_{6}
\end{array}\right]=\left[\begin{array}{c}
\omega_{m 1}^{2} \sin \left(\alpha_{s 1}\right) \\
\omega_{m 2}^{2} \sin \left(\alpha_{s 2}\right) \\
\omega_{m 3}^{2} \sin \left(\alpha_{s 3}\right) \\
\omega_{m 1}^{2} \cos \left(\alpha_{s 1}\right) \\
\omega_{m 2}^{2} \cos \left(\alpha_{s 2}\right) \\
\omega_{m 3}^{2} \cos \left(\alpha_{s 3}\right)
\end{array}\right],
$$

then the set of (24)-(27) can be written in the state-space form as

$$
\begin{aligned}
\dot{x}_{1}= & x_{2} x_{6}-x_{3} x_{5}+g \sin \left(x_{8}\right)-\frac{\sqrt{3} k_{f}}{2 m} u_{2}+\frac{\sqrt{3} k_{f}}{2 m} u_{3}, \\
\dot{x}_{2}= & x_{3} x_{4}-x_{1} x_{6}-g \sin \left(x_{7}\right) \cos \left(x_{8}\right) \\
& +\frac{k_{f}}{m} u_{1}-\frac{k_{f}}{2 m} u_{2}-\frac{k_{f}}{2 m} u_{3}, \\
\dot{x}_{3}= & x_{1} x_{5}-x_{2} x_{4}-g \cos \left(x_{7}\right) \cos \left(x_{8}\right) \\
& +\frac{k_{f}}{m} u_{4}+\frac{k_{f}}{m} u_{5}+\frac{k_{f}}{m} u_{6}, \\
\dot{x}_{4}= & \frac{x_{5} x_{6}\left(I_{y y}-I_{z z}\right)+I_{y z}\left(x_{5}^{2}-x_{6}^{2}\right)}{I_{x x}}+\frac{\sqrt{3} k_{d}}{2 I_{x x}} u_{2} \\
& -\frac{\sqrt{3} k_{d}}{2 I_{x x}} u_{3}+\frac{\sqrt{3} l k_{f}}{2 I_{x x}} u_{5}-\frac{\sqrt{3} l k_{f}}{2 I_{x x}} u_{6}, \\
\dot{x}_{5}= & \frac{x_{4} x_{5}\left(I_{x x} I_{y z}-I_{y y} I_{y z}-I_{z z} I_{y z}\right)}{I_{y y} I_{z z}-I_{y z}^{2}} \\
& +\frac{x_{4} x_{6}\left(I_{y z}^{2}+I_{z z}^{2}-I_{x x} I_{z z}\right)}{I_{y y} I_{z z}-I_{y z}^{2}}+\frac{I_{y z} k_{f} l-I_{z z} k_{d}}{I_{y y} I_{z z}-I_{y z}^{2}} u_{1} \\
& +\frac{2 I_{y z} k_{f} l+I_{z z} k_{d}}{2\left(I_{y y} I_{z z}-I_{y z}^{2}\right)} u_{2}+\frac{2 I_{y z} k_{f} l+I_{z z} k_{d}}{2\left(I_{y y} I_{z z}-I_{y z}^{2}\right)} u_{3} \\
& -\frac{I_{z z} k_{f} l+I_{y z} k_{d}}{I_{y y} I_{z z}-I_{y z}^{2}} u_{4}+\frac{I_{z z} k_{f} l-2 I_{y z} k_{d}}{2\left(I_{y y} I_{z z}-I_{y z}^{2}\right)} u_{5} \\
& +\frac{I_{z z} k_{f} l-2 I_{y z} k_{d}}{2\left(I_{y y} I_{z z}-I_{y z}^{2}\right)} u_{6},
\end{aligned}
$$




$$
\begin{aligned}
\dot{x}_{6}= & \frac{x_{4} x_{6}\left(I_{y y} I_{y z}+I_{z z} I_{y z}-I_{x x} I_{y z}\right)}{I_{y y} I_{z z}-I_{y z}^{2}} \\
& +\frac{x_{4} x_{5}\left(I_{x x} I_{y y}-I_{y z}^{2}-I_{y y}^{2}\right)}{I_{y y} I_{z z}-I_{y z}^{2}}+\frac{I_{y y} k_{f} l-I_{y z} k_{d}}{I_{y y} I_{z z}-I_{y z}^{2}} u_{1} \\
& +\frac{2 I_{y y} k_{f} l+I_{y z} k_{d}}{2\left(I_{y y} I_{z z}-I_{y z}^{2}\right)} u_{2}+\frac{2 I_{y y} k_{f} l+I_{y z} k_{d}}{2\left(I_{y y} I_{z z}-I_{y z}^{2}\right)} u_{3} \\
& -\frac{I_{y z} k_{f} l+I_{y y} k_{d}}{I_{y y} I_{z z}-I_{y z}^{2}} u_{4}+\frac{I_{y z} k_{f} l-2 I_{y y} k_{d}}{2\left(I_{y y} I_{z z}-I_{y z}^{2}\right)} u_{5} \\
& +\frac{I_{y z} k_{f} l-2 I_{y y} k_{d}}{2\left(I_{y y} I_{z z}-I_{y z}^{2}\right)} u_{6}, \\
\dot{x}_{7}= & x_{4}+x_{5} \sin \left(x_{7}\right) \tan \left(x_{8}\right)+x_{6} \cos \left(x_{7}\right) \tan \left(x_{8}\right), \\
\dot{x}_{8}= & x_{5} \cos \left(x_{7}\right)-x_{6} \sin \left(x_{7}\right), \\
\dot{x}_{9}= & x_{5} \sin \left(x_{7}\right) \sec \left(x_{8}\right)+x_{6} \cos \left(x_{7}\right) \sec \left(x_{8}\right), \\
\dot{x}_{10}= & x_{1} \cos \left(x_{8}\right) \cos \left(x_{9}\right)+x_{2}\left(\sin \left(x_{7}\right) \sin \left(x_{8}\right) \cos \left(x_{9}\right)\right. \\
& \left.-\cos \left(x_{7}\right) \sin \left(x_{9}\right)\right)+x_{3}\left(\cos \left(x_{7}\right) \sin \left(x_{8}\right) \cos \left(x_{9}\right)\right. \\
& \left.+\sin \left(x_{7}\right) \sin \left(x_{9}\right)\right), \\
\dot{x}_{11}= & x_{1} \cos \left(x_{8}\right) \sin \left(x_{9}\right)+x_{2}\left(\sin \left(x_{7}\right) \sin \left(x_{8}\right) \sin \left(x_{9}\right)\right. \\
& \left.+\cos \left(x_{7}\right) \cos \left(x_{9}\right)\right)+x_{3}\left(\cos \left(x_{7}\right) \sin \left(x_{8}\right) \sin \left(x_{9}\right)\right. \\
& \left.-\sin \left(x_{7}\right) \cos \left(x_{9}\right)\right), \\
& -x_{1} \sin \left(x_{8}\right)+x_{2} \sin \left(x_{7}\right) \cos \left(x_{8}\right) \\
& x_{3} \cos \left(x_{7}\right) \cos \left(x_{8}\right) \\
\dot{x}_{12} & \\
&
\end{aligned}
$$

The output vector is chosen as

$$
\boldsymbol{y}=\left[\begin{array}{c}
\phi \\
\theta \\
\psi \\
x_{v} \\
y_{v} \\
z_{v}
\end{array}\right]=\left[\begin{array}{c}
x_{7} \\
x_{8} \\
x_{9} \\
x_{10} \\
x_{11} \\
x_{12}
\end{array}\right] .
$$

Remark 1. Note that the real inputs $\left(\omega_{m i}\right.$ and $\left.\alpha_{s i}\right)$ are mapped into the control inputs $u_{i}$ via the nonlinear mapping (29). It can be shown that this nonlinear mapping is invertible thus giving actuator signals $\omega_{m i}$ and $\alpha_{s i}$ for use in real application. The physical actuator inputs $\omega_{m i}$ and $\alpha_{s i}$ can be calculated back from the control inputs $u_{i}$ via

$$
\alpha_{s i}=\arctan \left(\frac{u_{i}}{u_{i+3}}\right) \text { and } \omega_{m i}=\sqrt[4]{u_{i}^{2}+u_{i+3}^{2}} \forall i \in\{1,2,3\}
$$

\section{Control System Design}

The objective of this section is to design a robust distributed formation control protocol for swarms of the proposed tri-rotor UAV. Since the dynamical model of a single tri-rotor UAV is highly coupled and nonlinear, a robust feedback linearization technique is first applied to each tri-rotor to obtain simpler closed-loop dynamics. Then the swarm of identical tri-rotor UAVs is controlled through an optimal distributed formation control protocol which solves the time-varying formation tracking problem for tri-rotor robotic swarms.

\subsection{Robust Feedback Linearization}

Consider a single nonlinear system with $n$ states, $m$ inputs, and $m$ outputs described by

$$
\begin{gathered}
\dot{\boldsymbol{x}}=\boldsymbol{F}(\boldsymbol{x})+\boldsymbol{G}(\boldsymbol{x}) \boldsymbol{u}=\boldsymbol{F}(\boldsymbol{x})+\sum_{i=1}^{m} \boldsymbol{G}_{i}(\boldsymbol{x}) \boldsymbol{u}_{i}, \\
\boldsymbol{y}=\left[H_{1}(\boldsymbol{x}), \ldots, H_{m}(\boldsymbol{x})\right]^{T},
\end{gathered}
$$

where $\boldsymbol{x}(t) \in \mathbb{R}^{n}$ denotes the state vector, $\boldsymbol{u}(t) \in \mathbb{R}^{m}$ is the control input, $\boldsymbol{y}(t) \in \mathbb{R}^{m}$ is the output vector, and $\boldsymbol{F}(\boldsymbol{x}), \boldsymbol{G}_{1}(\boldsymbol{x}), \ldots$, $\boldsymbol{G}_{m}(\boldsymbol{x}), \boldsymbol{y}$ are smooth vector fields defined on an open subset of $\mathbb{R}^{n}$.

Suppose that this system satisfies the well-known conditions for feedback linearization [34]: The relative degree of $H_{i}$ is equal to $r_{i}$ for $i \in\{1, \ldots, m\}$ such that $r_{1}+\cdots+r_{m}=n$, and the decoupling matrix

$$
\boldsymbol{M}(\boldsymbol{x})=\left[\begin{array}{ccc}
L_{\boldsymbol{G}_{1}} L_{\boldsymbol{f}}^{r_{1}-1} H_{1}(\boldsymbol{x}) & \ldots & L_{\boldsymbol{G}_{m}} L_{\boldsymbol{f}}^{r_{1}-1} H_{1}(\boldsymbol{x}) \\
\vdots & \ddots & \vdots \\
L_{\boldsymbol{G}_{1}} L_{\boldsymbol{f}}^{r_{m}-1} H_{m}(\boldsymbol{x}) & \ldots & L_{\boldsymbol{G}_{m}} L_{\boldsymbol{f}}^{r_{m}-1} H_{m}(\boldsymbol{x})
\end{array}\right]
$$

is invertible, where $L_{(.)}($.) denotes the Lie derivative operator [34]. It is then possible to find a feedback linearizing control law of the form

$$
\boldsymbol{u}(\boldsymbol{x}, \boldsymbol{w})=\boldsymbol{\alpha}_{c}(\boldsymbol{x})+\boldsymbol{\beta}_{c}(\boldsymbol{x}) \boldsymbol{w},
$$

where $\boldsymbol{w}(t)$ is a new control input, and $\boldsymbol{\alpha}_{c}(\boldsymbol{x})=$ $-\boldsymbol{M}^{-1}(\boldsymbol{x})\left[\begin{array}{lll}L_{f}^{r_{1}} H_{1}(\boldsymbol{x}) & \ldots & L_{f}^{r_{m}} H_{m}(\boldsymbol{x})\end{array}\right]^{T}, \quad \boldsymbol{\beta}_{c}(\boldsymbol{x})=\boldsymbol{M}^{-1}(\boldsymbol{x})$, such that on application of the control law in (46), the nonlinear state-equation (43) reduces into the linear state-equation

$$
\dot{\boldsymbol{x}}_{c}=\boldsymbol{A}_{c} \boldsymbol{x}_{c}+\boldsymbol{B}_{c} \boldsymbol{w},
$$

where $\boldsymbol{A}_{c}$ and $\boldsymbol{B}_{c}$ are matrices of the Brunovsky canonical form [34], and a change of coordinates $\boldsymbol{x}_{c}=$ $\boldsymbol{\phi}_{c}(x)$ with $\boldsymbol{\phi}_{c}^{T}(\boldsymbol{x})=\left[\begin{array}{lll}\boldsymbol{\phi}_{c 1}^{T}(\boldsymbol{x}) & \ldots & \boldsymbol{\phi}_{c m}^{T}(\boldsymbol{x})\end{array}\right]$ and $\boldsymbol{\phi}_{c i}^{T}(\boldsymbol{x})=$ $\left[\begin{array}{llll}H_{i}(\boldsymbol{x}) & L_{f} H_{i}(\boldsymbol{x}) & \ldots & L_{f}^{r_{i}-1} H_{i}(\boldsymbol{x})\end{array}\right]$.

The robust feedback linearization technique [26], on the other hand, exactly transforms the nonlinear state-equation into a linear state-equation that is equal to the Jacobi linear approximation of the original nonlinear state-equation around the origin. This can then be controlled using linear techniques [27]. In the robust feedback linearization case, the linearized stateequation becomes

$$
\dot{\boldsymbol{x}}_{r}=\boldsymbol{A}_{r} \boldsymbol{x}_{r}+\boldsymbol{B}_{r} \boldsymbol{v}
$$

where $\boldsymbol{A}_{r}=\partial_{x} \boldsymbol{F}(\mathbf{0})$ and $\boldsymbol{B}_{r}=\boldsymbol{G}(\mathbf{0})$. The nonlinear stateequation (43) is geometrically transformed into the linear stateequation of any operating point, not only in a small neighborhood of the origin point. [26] argues that classical feedback linearization may be non robust in the presence of uncertainties as any system is transformed into a chain of integrators (i.e. Brunovsky form) whereas robust feedback linearization preserves some system information. 
The robust feedback linearization control law is

$$
u(x, v)=\alpha(x)+\beta(x) v,
$$

where

$$
\begin{gathered}
\boldsymbol{\alpha}(\boldsymbol{x})=\boldsymbol{\alpha}_{c}(\boldsymbol{x})+\boldsymbol{\beta}_{c}(\boldsymbol{x}) \boldsymbol{L} \boldsymbol{U}^{-1} \boldsymbol{\phi}_{c}(\boldsymbol{x}), \\
\boldsymbol{\beta}(\boldsymbol{x})=\boldsymbol{\beta}_{c}(\boldsymbol{x}) \boldsymbol{R}^{-1} \\
\boldsymbol{\phi}_{r}(\boldsymbol{x})=\boldsymbol{U}^{-1} \boldsymbol{\phi}_{c}(\boldsymbol{x}), \\
\boldsymbol{L}=-\boldsymbol{M}(\mathbf{0}) \partial_{\boldsymbol{x}} \boldsymbol{\alpha}_{c}(\mathbf{0}) \\
\boldsymbol{R}=\boldsymbol{M}^{-1}(\mathbf{0}) \\
\boldsymbol{U}=\partial_{\boldsymbol{x}} \boldsymbol{\phi}_{c}(\mathbf{0}) \\
\boldsymbol{x}_{r}=\boldsymbol{\phi}_{r}(\boldsymbol{x}) .
\end{gathered}
$$

Now we apply the robust feedback linearization to the dynamics of tri-rotor UAV system. The relative degrees are $r_{1}=2$, $r_{2}=2, r_{3}=2, r_{4}=2, r_{5}=2$ and $r_{6}=2$, resulting in a vector relative degree $r=12$, which is equal to the number of states. The decoupling matrix $\boldsymbol{M}(\boldsymbol{x})$ can also be written in a compact form as given in [6]:

$$
\boldsymbol{M}(\boldsymbol{x})=\left[\begin{array}{c}
\boldsymbol{\Psi}\left(\boldsymbol{I}_{v}^{b}\right)^{-1}\left(k_{f} \boldsymbol{H}_{t}-k_{d} \boldsymbol{H}_{f}\right) \\
\frac{k_{f}}{m} \boldsymbol{R}_{b}^{e} \boldsymbol{H}_{f}
\end{array}\right] .
$$

It can be verified that $\operatorname{det}[\boldsymbol{M}(\boldsymbol{x})] \neq 0$ as the pitch angle is assumed to be in the range of $-\pi / 2<\theta<\pi / 2$, such that $\boldsymbol{M}(\boldsymbol{x})$ is always invertible in this case. As a result, the conditions for feedback linearization are satisfied.

After calculating the classic Brunowski form linearizing input (46) and applying the formulas for the robust feeedback linearization (49)-(56), the system can then be robust feedback linearized into

$$
\begin{gathered}
\dot{\boldsymbol{x}}_{r}=\boldsymbol{A}_{r} \boldsymbol{x}_{r}+\boldsymbol{B}_{r} \boldsymbol{v}, \\
\boldsymbol{y}=\boldsymbol{C}_{r} \boldsymbol{x}_{r} .
\end{gathered}
$$

The state space matrix $\boldsymbol{A}_{r}, \boldsymbol{B}_{r}$ and $\boldsymbol{C}_{r}$ are shown at the bottom of the page.

Furthermore, $\boldsymbol{L}, \boldsymbol{R}$ and $\boldsymbol{U}$ are calculated by

$$
\begin{aligned}
& \boldsymbol{L}=\left[\begin{array}{cccccccccccc}
0 & 0 & 0 & 0 & 0 & 0 & 0 & 0 & 0 & 0 & 0 & 0 \\
0 & 0 & 0 & 0 & 0 & 0 & 0 & 0 & 0 & 0 & 0 & 0 \\
0 & 0 & 0 & 0 & 0 & 0 & 0 & 0 & 0 & 0 & 0 & 0 \\
0 & 0 & 0 & 0 & 0 & 0 & 0 & g & 0 & 0 & 0 & 0 \\
0 & 0 & 0 & 0 & 0 & 0 & -g & 0 & 0 & 0 & 0 & 0 \\
0 & 0 & 0 & 0 & 0 & 0 & 0 & 0 & 0 & 0 & 0 & 0
\end{array}\right], \\
& \boldsymbol{R}=\left[\begin{array}{rrrcrc}
0 & -\frac{I_{y z}}{3 k_{f} l} & \frac{I_{z z}}{3 k_{f} l} & 0 & \frac{2 m}{3 k_{f}} & \frac{k_{d} m}{3 k_{f}^{2} l} \\
0 & -\frac{I_{y z}}{3 k_{f} l} & \frac{I_{z z}}{3 f_{f} l} & -\frac{\sqrt{3} m}{3 k_{f}} & -\frac{m}{3 k_{f}} & \frac{k_{d} m}{3 k_{f}^{2} l} \\
0 & -\frac{I_{y z}}{3 k_{f} l} & \frac{I_{z z}}{3 k_{f} l} & \frac{\sqrt{3} m}{3 k_{f}} & -\frac{m}{3 k_{f}} & \frac{k_{d} m}{3 k_{f}^{2} l} \\
0 & -\frac{2 l_{y y}}{3 k_{f} l} & \frac{2 y_{z}}{3 k_{f} l} & 0 & -\frac{2 k_{d} m}{3 k_{f}^{2} l} & \frac{m}{3 k_{f}} \\
\frac{\sqrt{3} I_{x x}}{3 k_{f} l} & \frac{I_{y y}}{3 k_{f} l} & -\frac{I_{y z}}{3 k_{f} l} & \frac{\sqrt{3} k_{d} m}{3 k_{f}^{2} l} & \frac{k_{d} m}{3 k_{f}^{2} l} & \frac{m}{3 k_{f}} \\
-\frac{\sqrt{3} I_{x x}}{3 k_{f} l} & \frac{I_{y y}}{3 k_{f} l} & -\frac{I_{y z}}{3 k_{f} l} & -\frac{\sqrt{3} k_{d} m}{3 k_{f}^{2} l} & \frac{k_{d} m}{3 k_{f}^{2} l} & \frac{m}{3 k_{f}}
\end{array}\right],
\end{aligned}
$$

$$
\boldsymbol{U}=\left[\begin{array}{lllllllllllll}
0 & 0 & 0 & 0 & 0 & 0 & 1 & 0 & 0 & 0 & 0 & 0 \\
0 & 0 & 0 & 0 & 0 & 0 & 0 & 1 & 0 & 0 & 0 & 0 \\
0 & 0 & 0 & 0 & 0 & 0 & 0 & 0 & 0 & 1 & 0 & 0 & 0 \\
0 & 0 & 0 & 0 & 0 & 0 & 0 & 0 & 0 & 1 & 0 & 0 \\
0 & 0 & 0 & 0 & 0 & 0 & 0 & 0 & 0 & 0 & 0 & 1 & 0 \\
0 & 0 & 0 & 0 & 0 & 0 & 0 & 0 & 0 & 0 & 0 & 1 \\
0 & 0 & 0 & 1 & 0 & 0 & 0 & 0 & 0 & 0 & 0 & 0 \\
0 & 0 & 0 & 0 & 1 & 0 & 0 & 0 & 0 & 0 & 0 & 0 \\
0 & 0 & 0 & 0 & 0 & 1 & 0 & 0 & 0 & 0 & 0 & 0 \\
1 & 0 & 0 & 0 & 0 & 0 & 0 & 0 & 0 & 0 & 0 & 0 \\
0 & 1 & 0 & 0 & 0 & 0 & 0 & 0 & 0 & 0 & 0 \\
0 & 0 & 1 & 0 & 0 & 0 & 0 & 0 & 0 & 0 & 0 & 0
\end{array}\right],
$$

and $\phi_{c}$ and $\phi_{r}$ are given by

$$
\begin{aligned}
& \boldsymbol{\phi}_{c}=\left[\begin{array}{llllllllllll}
x_{7} & x_{8} & x_{9} & x_{10} & x_{11} & x_{12} & \dot{x}_{7} & \dot{x}_{8} & \dot{x}_{9} & \dot{x}_{10} & \dot{x}_{11} & \dot{x}_{12}
\end{array}\right]^{T},
\end{aligned}
$$

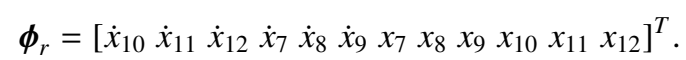

From (56) we know $\boldsymbol{x}_{r}=\phi_{r}(\boldsymbol{x})$. Finally, $\boldsymbol{\alpha}(\boldsymbol{x})$ and $\boldsymbol{\beta}(\boldsymbol{x})$ can then be computed from (50) and (51) directly.

\subsection{Distributed Optimal Formation Protocol Design}

In practical applications, some states do not need to be measured by sensors for controller design. For example, the vehicle's translational velocity in the body coordinate frame $\boldsymbol{v}_{v}^{b}$ is not used in the robust feedback linearization controller. It can hence be obtained by using an observer on input and output information of the feedback linearized system. In this section, we propose a distributed optimal formation protocol which uses the neighbourhood state estimation information for controller design and the local output estimation error information for the observer design. The scheme for controlling the dynamics of attitude and position of each tri-rotor UAV, based on robust feedback linearization and distributed optimal output feedback formation protocol, is illustrated in Fig. 4.

Consider a set of $N$ tri-rotor UAVs. Suppose that each trirotor UAV has the identical linearized dynamics described by

$$
\begin{gathered}
\dot{\boldsymbol{x}}_{r i}=\boldsymbol{A}_{r} \boldsymbol{x}_{r i}+\boldsymbol{B}_{r} \boldsymbol{v}_{i}, \\
\boldsymbol{y}_{i}=\boldsymbol{C}_{r} \boldsymbol{x}_{r i} .
\end{gathered}
$$

It can be easily verified that $\left(\boldsymbol{A}_{r}, \boldsymbol{B}_{r}, \boldsymbol{C}_{r}\right)$ is stabilizable and detectable.

The dynamics of the leader node, labeled 0 , is given by

$$
\dot{\boldsymbol{x}}_{0}=\boldsymbol{A}_{r} \boldsymbol{x}_{0}
$$

$$
\boldsymbol{y}_{0}=\boldsymbol{C}_{r} \boldsymbol{x}_{0}
$$

where $\boldsymbol{x}_{0} \in \mathbb{R}^{n}$ is the state, $\boldsymbol{y}_{0} \in \mathbb{R}^{p}$ is the output. It can be considered as a command generator, which generates the desired target trajectory. The leader can be observed from a subset of agents in a graph. If node $i$ observes the leader, an edge $(0, i)$ is said to exist with weighting gain $g_{i}>0$ as a pinned node. We denote the pinning matrix as $\boldsymbol{G}=\operatorname{diag}\left\{g_{i}\right\} \in \mathbb{R}^{N \times N}$.

The desired formation is specified by the vector $\boldsymbol{h}=$ $\left[\boldsymbol{h}_{1}^{T}, \boldsymbol{h}_{2}^{T}, \ldots, \boldsymbol{h}_{N}^{T}\right]^{T}$ with $\boldsymbol{h}_{i} \in \mathbb{R}^{n}$ being a preset vector known by the corresponding $i^{t h}$ agent. It should be noted that the formation problem reduces to a consensus problem when $\boldsymbol{h}_{i}=\mathbf{0} \forall$ $i \in\{1, \ldots, N\}$. 


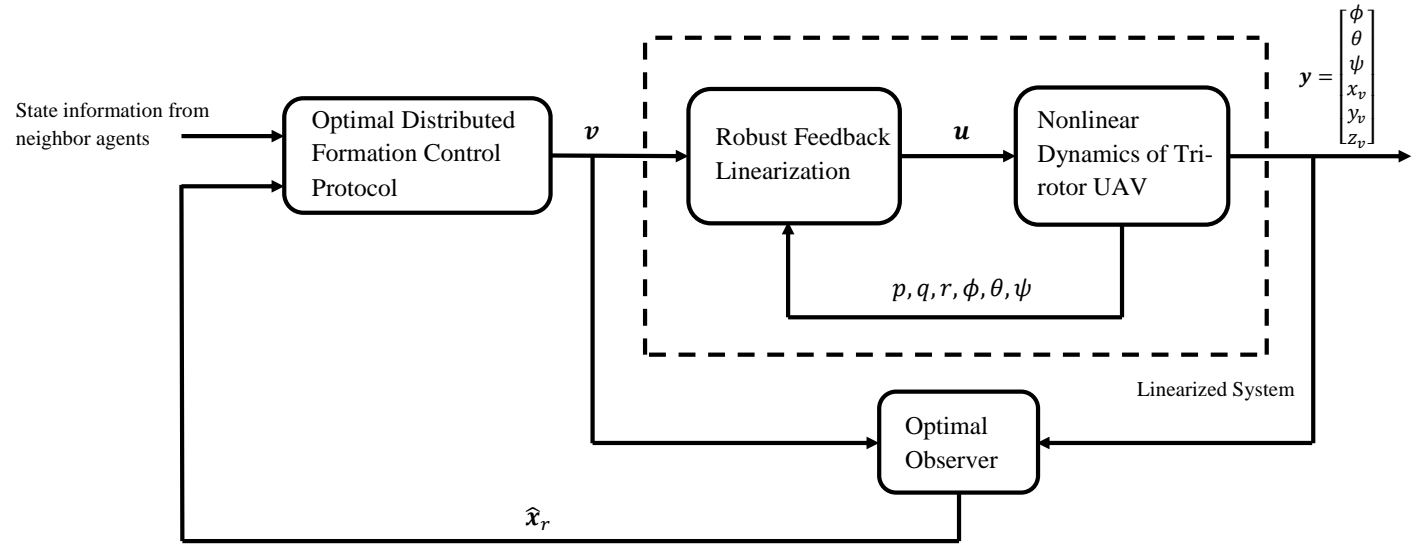

Fig. 4. Control system scheme: Distributed optimal formation control law and robust feedback linearization combining linear and nonlinear parts

Denote the estimate of the state $\boldsymbol{x}_{r i}$ by $\hat{\boldsymbol{x}}_{r i} \in \mathbb{R}^{n}$ and let the state estimation error be $\tilde{\boldsymbol{x}}_{r i}=\boldsymbol{x}_{r i}-\hat{\boldsymbol{x}}_{r i}$. Then the consequent estimate of the output $\boldsymbol{y}_{i}$ is given by $\hat{\boldsymbol{y}}_{i}=\boldsymbol{C}_{r} \hat{\boldsymbol{x}}_{r i}$ and the output estimation error for node $i$ is given by $\tilde{\boldsymbol{y}}_{i}=\boldsymbol{y}_{i}-\hat{\boldsymbol{y}}_{i}$. Consider the following distributed optimal formation protocol

$$
\begin{gathered}
\boldsymbol{v}_{i}=c \boldsymbol{K} \sum_{j \in N_{i}} a_{i j}\left(\left(\hat{\boldsymbol{x}}_{r j}-\boldsymbol{h}_{j}\right)-\left(\hat{\boldsymbol{x}}_{r i}-\boldsymbol{h}_{i}\right)\right) \\
+c \boldsymbol{K} g_{i}\left(\boldsymbol{x}_{0}-\left(\hat{\boldsymbol{x}}_{r i}-\boldsymbol{h}_{i}\right)\right)+\boldsymbol{\gamma}_{i}, \\
\dot{\boldsymbol{x}}_{r i}=\boldsymbol{A}_{r} \hat{\boldsymbol{x}}_{r i}+\boldsymbol{B}_{r} \boldsymbol{v}_{i}-c \boldsymbol{F} \tilde{\boldsymbol{y}}_{i},
\end{gathered}
$$

where $c>0$ is the scalar coupling gain, $\boldsymbol{K} \in \mathbb{R}^{m \times n}$ is the feedback control gain matrix, $\boldsymbol{F} \in \mathbb{R}^{n \times m}$ is the observer gain, and $\gamma_{i} \in \mathbb{R}^{m}$ represents the formation compensation signal to be designed.
Let $\boldsymbol{x}=\left[\boldsymbol{x}_{r 1}^{T}, \boldsymbol{x}_{r 2}^{T}, \ldots, \boldsymbol{x}_{r N}^{T}\right]^{T}, \hat{\boldsymbol{x}}=\left[\hat{\boldsymbol{x}}_{r 1}^{T}, \hat{\boldsymbol{x}}_{r 2}^{T}, \ldots, \hat{\boldsymbol{x}}_{r N}^{T}\right]^{T}, \tilde{\boldsymbol{x}}=$ $\left[\tilde{\boldsymbol{x}}_{r 1}^{T}, \tilde{\boldsymbol{x}}_{r 2}^{T}, \ldots, \tilde{\boldsymbol{x}}_{r N}^{T}\right]^{T}, \underline{\boldsymbol{x}}_{0}=\mathbf{1}_{N} \otimes \boldsymbol{x}_{0}$, and $\boldsymbol{\gamma}=\left[\boldsymbol{\gamma}_{1}^{T}, \boldsymbol{\gamma}_{2}^{T}, \ldots, \boldsymbol{\gamma}_{N}^{T}\right]^{T}$. Under a control protocol with directed topology, the tri-rotor UAV swarm can be written in a compact form as

$$
\begin{aligned}
\dot{\boldsymbol{x}}= & \left(\boldsymbol{I}_{N} \otimes \boldsymbol{A}_{r}\right) \boldsymbol{x}-c\left[(\boldsymbol{L}+\boldsymbol{G}) \otimes \boldsymbol{B}_{r} K\right]\left(\hat{\boldsymbol{x}}-\underline{\boldsymbol{x}}_{0}\right) \\
& +c\left[(\boldsymbol{L}+\boldsymbol{G}) \otimes \boldsymbol{B}_{r} \boldsymbol{K}\right] \boldsymbol{h}+\left(\boldsymbol{I}_{N} \otimes \boldsymbol{B}_{r}\right) \boldsymbol{\gamma}, \\
\dot{\hat{\boldsymbol{x}}}= & \left(\boldsymbol{I}_{N} \otimes \boldsymbol{A}_{r}\right) \hat{\boldsymbol{x}}-\left(\boldsymbol{I}_{N} \otimes c \boldsymbol{F} \boldsymbol{C}_{r}\right)(\boldsymbol{x}-\hat{\boldsymbol{x}}) \\
& -c\left[(\boldsymbol{L}+\boldsymbol{G}) \otimes \boldsymbol{B}_{r} \boldsymbol{K}\right]\left(\hat{\boldsymbol{x}}-\underline{\boldsymbol{x}}_{0}\right) \\
& +c\left[(\boldsymbol{L}+\boldsymbol{G}) \otimes \boldsymbol{B}_{r} \boldsymbol{K}\right] \boldsymbol{h}+\left(\boldsymbol{I}_{N} \otimes \boldsymbol{B}_{r}\right) \boldsymbol{\gamma} .
\end{aligned}
$$

It follows the fact that matrix $\boldsymbol{B}_{r}$ given in (61) is of full rank, there always exists a nonsingular matrix $\left[\tilde{\boldsymbol{B}}^{T}, \overline{\boldsymbol{B}}^{T}\right]^{T}$ with $\tilde{\boldsymbol{B}} \in$

$$
\begin{aligned}
& \boldsymbol{A}_{r}=\left[\begin{array}{cccccccccccc}
0 & 0 & 0 & 0 & 0 & 0 & 0 & g & 0 & 0 & 0 & 0 \\
0 & 0 & 0 & 0 & 0 & 0 & -g & 0 & 0 & 0 & 0 & 0 \\
0 & 0 & 0 & 0 & 0 & 0 & 0 & 0 & 0 & 0 & 0 & 0 \\
0 & 0 & 0 & 0 & 0 & 0 & 0 & 0 & 0 & 0 & 0 & 0 \\
0 & 0 & 0 & 0 & 0 & 0 & 0 & 0 & 0 & 0 & 0 & 0 \\
0 & 0 & 0 & 0 & 0 & 0 & 0 & 0 & 0 & 0 & 0 & 0 \\
0 & 0 & 0 & 1 & 0 & 0 & 0 & 0 & 0 & 0 & 0 & 0 \\
0 & 0 & 0 & 0 & 1 & 0 & 0 & 0 & 0 & 0 & 0 & 0 \\
0 & 0 & 0 & 0 & 0 & 1 & 0 & 0 & 0 & 0 & 0 & 0 \\
1 & 0 & 0 & 0 & 0 & 0 & 0 & 0 & 0 & 0 & 0 & 0 \\
0 & 1 & 0 & 0 & 0 & 0 & 0 & 0 & 0 & 0 & 0 & 0 \\
0 & 0 & 1 & 0 & 0 & 0 & 0 & 0 & 0 & 0 & 0 & 0
\end{array}\right], \\
& \boldsymbol{B}_{r}=\left[\begin{array}{cccccc}
0 & -\frac{\sqrt{3} k_{f}}{2 m} & \frac{\sqrt{3} k_{f}}{2 m} & 0 & 0 & 0 \\
\frac{k_{f}}{m} & -\frac{k_{f}}{2 m} & -\frac{k_{f}}{2 m} & 0 & 0 & 0 \\
0 & 0 & 0 & \frac{k_{f}}{m} & \frac{k_{f}}{m} & \frac{k_{f}}{m} \\
0 & \frac{\sqrt{3} k_{d}}{2 I_{x x}} & -\frac{\sqrt{3} k_{d}}{2 I_{x x}} & 0 & \frac{\sqrt{3} k_{f}}{2 I_{x x}} & -\frac{\sqrt{3} k_{f}}{2 I_{x x}} \\
\frac{I_{y z} k_{f} l-I_{z z} k_{d}}{I_{y y} I_{z z}-I_{y z}^{2}} & \frac{2 I_{y z} k_{f} l+I_{z z} k_{d}}{2\left(I_{y y} I_{z z}-I_{y z}^{2}\right)} & \frac{2 I_{y z} k_{f} l+I_{z z} k_{d}}{2\left(I_{y y} I_{z z}-I_{y z}^{2}\right)} & -\frac{I_{z z} k_{f} l+I_{y z} k_{d}}{I_{y y} I_{z z}-I_{y z}^{2}} & \frac{I_{z z} k_{f} l-2 I_{y z} k_{d}}{2\left(I_{y y} I_{z z}-I_{y z}^{2}\right)} & \frac{I_{z z} k_{f} l-2 I_{y z} k_{d}}{2\left(I_{y y} I_{z z}-I_{y z}^{2}\right)} \\
\frac{I_{y y} k_{f} l-I_{y z} k_{d}}{I_{y y} I_{z z}-I_{y z}^{2}} & \frac{2 I_{y y} k_{f} l+I_{y z} k_{d}}{2\left(I_{y y} I_{z z}-I_{y z}^{2}\right)} & \frac{2 I_{y y} k_{f} l+I_{y z} k_{d}}{2\left(I_{y y} I_{z z}-I_{y z}^{2}\right)} & -\frac{I_{y z} k_{f} l+I_{y y} k_{d}}{I_{y y} I_{z z}-I_{y z}^{2}} & \frac{I_{y z} k_{f}-2 I_{y y} k_{d}}{2\left(I_{y y} I_{z z}-I_{y z}^{2}\right)} & \frac{I_{y z} k_{f} l-2 I_{y y} k_{d}}{2\left(I_{y y} I_{z z}-I_{y z}^{2}\right)} \\
0 & 0 & 0 & 0 & 0 & 0 \\
0 & 0 & 0 & 0 & 0 & 0 \\
0 & 0 & 0 & 0 & 0 & 0 \\
0 & 0 & 0 & 0 & 0 & 0 \\
0 & 0 & 0 & 0 & 0 & 0
\end{array}\right], \\
& \boldsymbol{C}_{r}=\left[\begin{array}{lllllllllllll}
0 & 0 & 0 & 0 & 0 & 0 & 1 & 0 & 0 & 0 & 0 & 0 \\
0 & 0 & 0 & 0 & 0 & 0 & 0 & 1 & 0 & 0 & 0 & 0 \\
0 & 0 & 0 & 0 & 0 & 0 & 0 & 0 & 1 & 0 & 0 & 0 \\
0 & 0 & 0 & 0 & 0 & 0 & 0 & 0 & 0 & 1 & 0 & 0 \\
0 & 0 & 0 & 0 & 0 & 0 & 0 & 0 & 0 & 0 & 1 & 0 \\
0 & 0 & 0 & 0 & 0 & 0 & 0 & 0 & 0 & 0 & 0 & 1
\end{array}\right] .
\end{aligned}
$$


$\mathbb{R}^{m \times n}$ and $\overline{\boldsymbol{B}} \in \mathbb{R}^{(n-m) \times n}$ such that $\tilde{\boldsymbol{B}} \boldsymbol{B}_{r}=\boldsymbol{I}_{m}$ and $\overline{\boldsymbol{B}} \boldsymbol{B}_{r}=\mathbf{0}$. The following theorem which is motivated by [35], has been improved to deal with output feedback tracking of multi-agent systems.

Theorem 1. Let $\lambda_{i}(i \in\{1, \ldots, N\})$ be the eigenvalues of $(\boldsymbol{L}+\boldsymbol{G})$. Then the tri-rotor UAV swarm with directed interaction topology asymptotically converges to the formation specified by $\left(\boldsymbol{x}_{0}+\boldsymbol{h}_{i}\right) \in \mathbb{R}^{n} \forall i \in\{1, \ldots, N\}$ if the following conditions hold for all $i \in\{1, \ldots, N\}$

$$
\overline{\boldsymbol{B}} \boldsymbol{A}_{r} \boldsymbol{h}_{i}-\overline{\boldsymbol{B}} \dot{\boldsymbol{h}}_{i}=\mathbf{0},
$$

$$
\boldsymbol{A}_{r}-c \lambda_{i} \boldsymbol{B}_{r} \boldsymbol{K} \text { and } \boldsymbol{A}_{r}+c \boldsymbol{F} \boldsymbol{C}_{r} \text { are Hurwitz, }
$$

$$
\text { and } \boldsymbol{\gamma}_{i}=\tilde{\boldsymbol{B}} \dot{\boldsymbol{h}}_{i}-\tilde{\boldsymbol{B}} \boldsymbol{A}_{r} \boldsymbol{h}_{i} \text { for all } i \in\{1, \ldots, N\} \text {. }
$$

Proof. Let formation tracking error for each UAV be $\boldsymbol{\Phi}_{i}=$ $\boldsymbol{x}_{r i}-\boldsymbol{h}_{i}-\boldsymbol{x}_{0}$ and $\boldsymbol{\Phi}=\left[\boldsymbol{\Phi}_{1}^{T}, \boldsymbol{\Phi}_{2}^{T}, \ldots, \boldsymbol{\Phi}_{N}^{T}\right]^{T}$. Then the global formation error dynamics with directed interaction topology can be written as

$$
\begin{aligned}
\dot{\boldsymbol{\Phi}}= & {\left[\boldsymbol{I}_{N} \otimes \boldsymbol{A}_{r}-c(\boldsymbol{L}+\boldsymbol{G}) \otimes \boldsymbol{B}_{r} \boldsymbol{K}\right] \boldsymbol{\Phi} } \\
& +c\left[(\boldsymbol{L}+\boldsymbol{G}) \otimes \boldsymbol{B}_{r} \boldsymbol{K}\right] \tilde{\boldsymbol{x}} \\
& +\left(\boldsymbol{I}_{N} \otimes \boldsymbol{A}_{r}\right) \boldsymbol{h}-\left(\boldsymbol{I}_{N} \otimes \boldsymbol{I}_{N}\right) \dot{\boldsymbol{h}} \\
& +\left(\boldsymbol{I}_{N} \otimes \boldsymbol{B}_{r}\right) \boldsymbol{\gamma} .
\end{aligned}
$$

The global observer error dynamics is

$$
\dot{\tilde{\boldsymbol{x}}}=\boldsymbol{I}_{N} \otimes\left(\boldsymbol{A}_{r}+c \boldsymbol{F} \boldsymbol{C}_{r}\right) \tilde{\boldsymbol{x}} .
$$

In view of Assumption 1, all the eigenvalues of matrix $(\boldsymbol{L}+$ $\boldsymbol{G})$ have positive real parts [36]. It is well known that there exists a nonsingular $\boldsymbol{T}$ such that $\boldsymbol{T}^{-1}(\boldsymbol{L}+\boldsymbol{G}) \boldsymbol{T}$ is in the Jordan canonical form $\boldsymbol{J}$. Let $\boldsymbol{\vartheta}=\left(\boldsymbol{T}^{-1} \otimes \boldsymbol{I}_{n}\right) \boldsymbol{\Phi}=\left[\boldsymbol{\vartheta}_{1}^{T}, \boldsymbol{\vartheta}_{2}^{T}, \ldots, \boldsymbol{\vartheta}_{N}^{T}\right]^{T}$. Then multi-agent system can be represented in terms of $\boldsymbol{\vartheta}$ as

$$
\begin{aligned}
\dot{\boldsymbol{\vartheta}}= & \left(\boldsymbol{I}_{N} \otimes \boldsymbol{A}_{r}-c \boldsymbol{J} \otimes \boldsymbol{B}_{r} \boldsymbol{K}\right) \boldsymbol{\vartheta} \\
& +c\left[\boldsymbol{T}^{-1}(\boldsymbol{L}+\boldsymbol{G}) \otimes \boldsymbol{B}_{r} \boldsymbol{K}\right] \tilde{\boldsymbol{x}} \\
& +\left(\boldsymbol{T}^{-1} \otimes \boldsymbol{A}_{r}\right) \boldsymbol{h}-\left(\boldsymbol{T}^{-1} \otimes \boldsymbol{I}_{N}\right) \boldsymbol{h} \\
& +\left(\boldsymbol{T}^{-1} \otimes \boldsymbol{B}_{r}\right) \boldsymbol{\gamma} .
\end{aligned}
$$

If condition (76) holds, then for all $i \in\{1, \ldots, N\}$

$$
\overline{\boldsymbol{B}} \boldsymbol{A}_{r} \boldsymbol{h}_{i}-\overline{\boldsymbol{B}} \dot{\boldsymbol{h}}_{i}+\overline{\boldsymbol{B}} \boldsymbol{B}_{r} \gamma_{i}=\mathbf{0} .
$$

By letting $\boldsymbol{\gamma}_{i}=\tilde{\boldsymbol{B}} \dot{\boldsymbol{h}}_{i}-\tilde{\boldsymbol{B}} \boldsymbol{A}_{r} \boldsymbol{h}_{i}$, it follows that

$$
\tilde{\boldsymbol{B}} \boldsymbol{A}_{r} \boldsymbol{h}_{i}-\tilde{\boldsymbol{B}} \dot{\boldsymbol{h}}_{i}+\tilde{\boldsymbol{B}} \boldsymbol{B}_{r} \gamma_{i}=\mathbf{0} .
$$

From (82) and (83) and the fact that $\left[\tilde{\boldsymbol{B}}^{T}, \overline{\boldsymbol{B}}^{T}\right]^{T}$ is nonsingular, one gets

$$
\boldsymbol{A}_{r} \boldsymbol{h}_{i}-\dot{\boldsymbol{h}}_{i}+\boldsymbol{B}_{r} \boldsymbol{\gamma}_{i}=\mathbf{0}
$$

which means that

$$
\left(\boldsymbol{I}_{N} \otimes \boldsymbol{A}_{r}\right) \boldsymbol{h}-\left(\boldsymbol{I}_{N} \otimes \boldsymbol{I}_{N}\right) \dot{\boldsymbol{h}}+\left(\boldsymbol{I}_{N} \otimes \boldsymbol{B}_{r}\right) \boldsymbol{\gamma}=\mathbf{0} .
$$

Pre-multiplying the both sides of (85) by $\boldsymbol{T}^{-1} \otimes \boldsymbol{I}_{N}$ yields

$$
\left(\boldsymbol{T}^{-1} \otimes \boldsymbol{A}_{r}\right) \boldsymbol{h}-\left(\boldsymbol{T}^{-1} \otimes \boldsymbol{I}_{N}\right) \dot{\boldsymbol{h}}+\left(\boldsymbol{T}^{-1} \otimes \boldsymbol{B}_{r}\right) \boldsymbol{\gamma}=\mathbf{0} .
$$

Then (81) reduces to the following dynamics

$$
\dot{\boldsymbol{\vartheta}}=\left(\boldsymbol{I}_{N} \otimes \boldsymbol{A}_{r}-c \boldsymbol{J} \otimes \boldsymbol{B}_{r} \boldsymbol{K}\right) \boldsymbol{\vartheta}+c\left[\boldsymbol{T}^{-1}(\boldsymbol{L}+\boldsymbol{G}) \otimes \boldsymbol{B}_{r} \boldsymbol{K}\right] \tilde{\boldsymbol{x}} .
$$

From (87) and (80), it can be obtained that

$$
\left[\begin{array}{c}
\dot{\boldsymbol{\vartheta}} \\
\dot{\tilde{\boldsymbol{x}}}
\end{array}\right]=\left[\begin{array}{cc}
\boldsymbol{A}_{e} & \boldsymbol{B}_{e} \\
\mathbf{0} & \boldsymbol{I}_{N} \otimes\left(\boldsymbol{A}_{r}+c \boldsymbol{F} \boldsymbol{C}_{r}\right)
\end{array}\right]\left[\begin{array}{l}
\boldsymbol{\vartheta} \\
\tilde{\boldsymbol{x}}
\end{array}\right],
$$

where

$$
\begin{aligned}
& \boldsymbol{A}_{e}=\boldsymbol{I}_{N} \otimes \boldsymbol{A}_{r}-c \boldsymbol{J} \otimes \boldsymbol{B}_{r} \boldsymbol{K}, \\
& \boldsymbol{B}_{e}=c \boldsymbol{T}^{-1}(\boldsymbol{L}+\boldsymbol{G}) \otimes \boldsymbol{B}_{r} \boldsymbol{K} .
\end{aligned}
$$

Therefore, the global error system in (88) is asymptotically stable if and only if both $\boldsymbol{A}_{e}$ and $\boldsymbol{I}_{N} \otimes\left(\boldsymbol{A}_{r}+c \boldsymbol{F} \boldsymbol{C}_{r}\right)$ are Hurwitz, and the latter can be satisfied due to the detectability of $\left(\boldsymbol{C}_{r}, \boldsymbol{A}_{r}\right)$. Note that the the state matrix $\boldsymbol{A}_{e}$ is either block diagonal or block upper-triangular. Hence the stability of (88) is equivalent to the stability of the $N$ subsystems defined with the diagonal blocks. Therefore, $\boldsymbol{A}_{r}-c \lambda_{i} \boldsymbol{B}_{r} \boldsymbol{K}$ is Hurwitz $\forall i \in\{1, \ldots, N\}$ if and only if $\boldsymbol{I}_{N} \otimes \boldsymbol{A}_{r}-c \boldsymbol{J} \otimes \boldsymbol{B}_{r} \boldsymbol{K}$ is Hurwitz. Therefore, $\boldsymbol{\vartheta}$ converges asymptotically to the origin which is equivalent to stating that $\boldsymbol{x}_{r i}$ converges asymptotically to $\boldsymbol{x}_{0}+\boldsymbol{h}_{i}$ for all $i \in\{1, \ldots, N\}$.

Next we will show how to select state variable feedback control gain $\boldsymbol{K}$ to guarantee stability on arbitrary directed graphs containing a spanning tree by using LQR based optimal design and proper choice of the coupling gain $c$. The following theorem is an extension of a result in [28], which only considers the consensus problem. In the case where $\boldsymbol{h}=\mathbf{0}$, the optimal formation tracking protocol (72) becomes the optimal consensus tracking protocol of [28], so it can be viewed as a special case of the result in the current paper.

Theorem 2. Let $\boldsymbol{Q}=\boldsymbol{Q}^{T} \in \mathbb{R}^{n \times n}$ and $\boldsymbol{R}=\boldsymbol{R}^{T} \in \mathbb{R}^{m \times m}$ be positive definite matrices. Let $\boldsymbol{P}$ be the unique positive definite solution of the algebraic Riccati equation

$$
\boldsymbol{A}_{r}^{T} \boldsymbol{P}+\boldsymbol{P} \boldsymbol{A}_{r}+\boldsymbol{Q}-\boldsymbol{P} \boldsymbol{B}_{r} \boldsymbol{R}^{-1} \boldsymbol{B}_{r}^{T} \boldsymbol{P}=\mathbf{0} .
$$

Then, under Assumption 1 and condition (76), the distributed formation tracking control protocol (72) with

$$
\boldsymbol{K}=\boldsymbol{R}^{-1} \boldsymbol{B}_{r}^{T} \boldsymbol{P}
$$

and $\gamma_{i}$ set as in (78) ensures that the tri-rotor UAV swarm with directed interaction topology asymptotically converges to the formation specified by $\left(\boldsymbol{x}_{0}+\boldsymbol{h}_{i}\right) \in \mathbb{R}^{n} \forall i \in\{1, \ldots, N\}$ if the coupling gain

$$
c \geq \frac{1}{2 \lambda_{R}}
$$

with $\lambda_{R}=\min _{i \in\{1, \ldots, N\}} \operatorname{Re}\left(\lambda_{i}\right)$, where $\lambda_{i}$ are the eigenvalues of $(\boldsymbol{L}+\boldsymbol{G})$. 
Proof. Consider the stability of the following subsystem

$$
\dot{\boldsymbol{\delta}}_{i}=\left(\boldsymbol{A}_{r}-c \lambda_{i} \boldsymbol{B}_{r} \boldsymbol{K}\right) \boldsymbol{\delta}_{i},
$$

where $\delta$ denotes the formation tracking closed-loop error. Construct the following Lyapunov candidate function

$$
V_{i}=\boldsymbol{\delta}_{i}^{*} \boldsymbol{P} \boldsymbol{\delta}_{i}
$$

Taking the derivative of $V_{i}$ along the trajectory of subsystem gives

$$
\dot{V}_{i}=\boldsymbol{\delta}_{i}^{*}\left(\boldsymbol{P} \boldsymbol{A}_{r}+\boldsymbol{A}_{r}^{T} \boldsymbol{P}-c \lambda_{i}^{*}\left(\boldsymbol{B}_{r} \boldsymbol{K}\right)^{T} \boldsymbol{P}-c \lambda_{i} \boldsymbol{P} \boldsymbol{B}_{r} \boldsymbol{K}\right) \boldsymbol{\delta}_{i} .
$$

Substituting $\boldsymbol{K}=\boldsymbol{R}^{-1} \boldsymbol{B}_{r}^{T} \boldsymbol{P}$ and $\boldsymbol{A}_{r}^{T} \boldsymbol{P}+\boldsymbol{P} \boldsymbol{A}_{r}=-\boldsymbol{Q}+\boldsymbol{P} \boldsymbol{B}_{r} \boldsymbol{R}^{-1} \boldsymbol{B}_{r}^{T} \boldsymbol{P}$ into (92) one has

$$
\dot{V}_{i}=\left[1-2 c \operatorname{Re}\left(\lambda_{i}\right)\right] \boldsymbol{\delta}_{i}^{*}\left(\boldsymbol{P} B_{r} \boldsymbol{R}^{-1} \boldsymbol{B}_{r}^{T} \boldsymbol{P}\right) \boldsymbol{\delta}_{i}-\boldsymbol{\delta}_{i}^{*} \boldsymbol{Q} \boldsymbol{\delta}_{i}
$$

It can be seen that if condition (91) holds, then $\dot{V}_{i}<0$. Therefore, $\boldsymbol{A}_{r}-c \lambda_{i} \boldsymbol{B}_{r} \boldsymbol{K}$ is Hurwitz for all $i \in\{1, \ldots, N\}$ by Lyapunov theory [37]. This completes the proof.

The ARE in (89) is extracted by minimizing the following performance index for each tri-rotor UAV

$$
J_{i}=\frac{1}{2} \int_{0}^{\infty}\left(\boldsymbol{\delta}_{i}^{T} \boldsymbol{Q} \boldsymbol{\delta}_{i}+\boldsymbol{v}_{i}^{T} \boldsymbol{R} \boldsymbol{v}_{i}\right) d t .
$$

The design Riccati matrices $\boldsymbol{Q}$ and $\boldsymbol{R}$ can be selected to adjust the relative cost of formation tracking error and control effort. This allows the cooperative control system to be tuned to tradeoff between the speed of formation tracking and the speed of DC motors to achieve it.

Remark 2. In order to enhance the robustness of our tri-rotor $\mathrm{UAV}$, suppose external white noises $\boldsymbol{\varepsilon}_{1}$ and $\boldsymbol{\varepsilon}_{2}$ are added to (68) and (69) respectively, which satisfy that

$$
E\left[\varepsilon_{1} \varepsilon_{1}^{T}\right]=\overline{\boldsymbol{Q}}, E\left[\boldsymbol{\varepsilon}_{2} \boldsymbol{\varepsilon}_{2}^{T}\right]=\overline{\boldsymbol{R}}, E\left[\boldsymbol{\varepsilon}_{1} \boldsymbol{\varepsilon}_{2}^{T}\right]=\mathbf{0},
$$

where $E$ donates the expected value, and $\overline{\boldsymbol{Q}}$ and $\overline{\boldsymbol{R}}$ are positive definite matrices. Then a local optimal observer gain $\boldsymbol{F}$ can be calculated by a similar approach (see [38] for further details) as

$$
\boldsymbol{F}=\overline{\boldsymbol{P}} \boldsymbol{C}_{r}^{T} \overline{\boldsymbol{R}}^{-1},
$$

where $\overline{\boldsymbol{P}}$ is the unique positive definite solution of ARE

$$
\boldsymbol{A}_{r} \overline{\boldsymbol{P}}+\overline{\boldsymbol{P}} \boldsymbol{A}_{r}^{T}-\overline{\boldsymbol{P}} \boldsymbol{C}_{r}^{T} \overline{\boldsymbol{R}}^{-1} \boldsymbol{C}_{r} \overline{\boldsymbol{P}}+\overline{\boldsymbol{Q}}=\mathbf{0} .
$$

This optimal observer is also known as Kalman-Bucy filter [39], which has been widely used in system state estimation. It has been demonstrated [40] to have many advantages, including optimality of state estimation in the presence of white noise and external disturbance [41].

With the above analysis, the procedure to construct the control law $\boldsymbol{u}_{i}$ is given in Algorithm 1.

Validation of internal stability using closed-loop data from experiments can be performed using technique described in [42]. This is useful as one would also expect unmodelled dynamics.
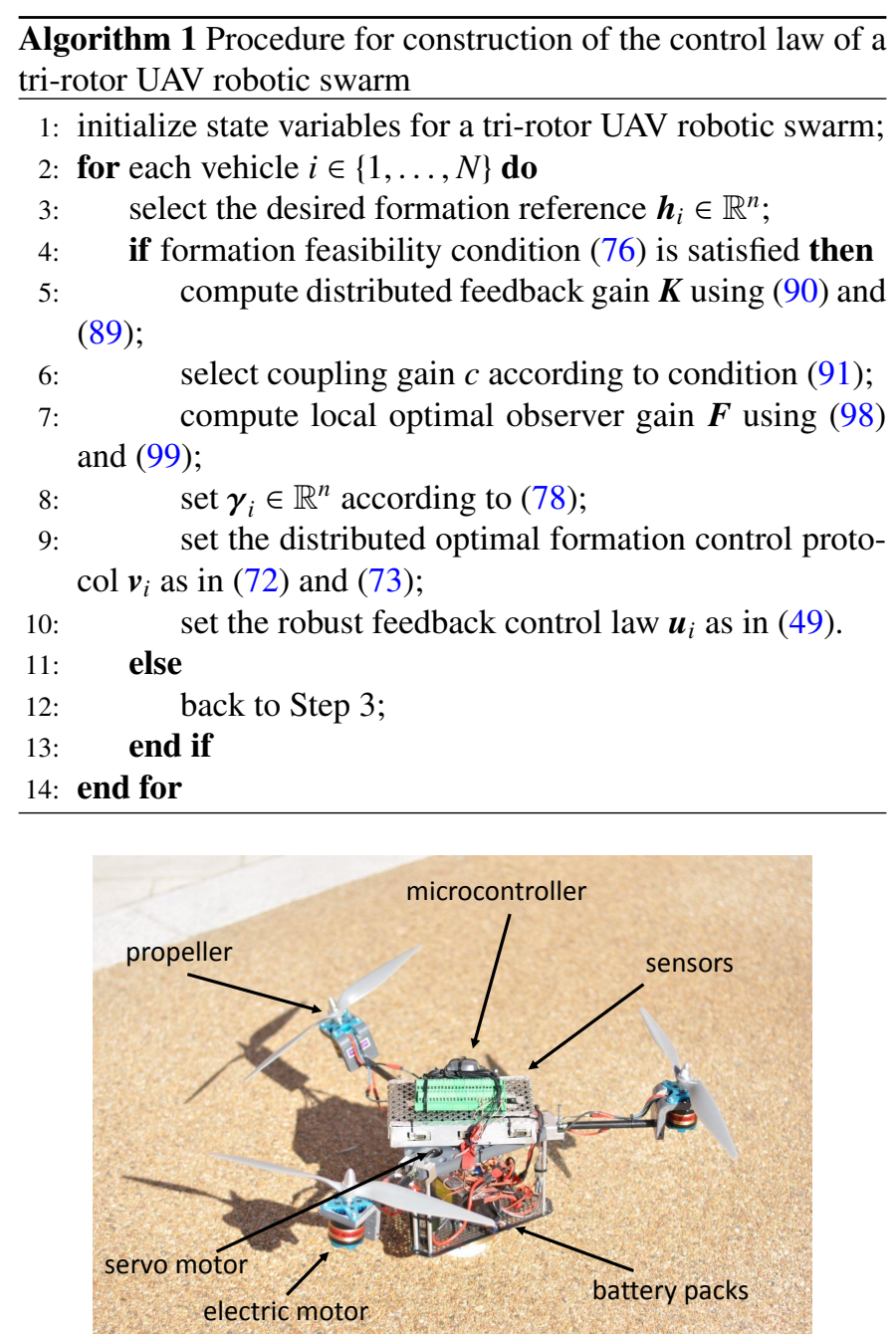

Fig. 5. The hardware of the designed tri-rotor UAV

\section{Simulation Results}

The numerical simulation carried out in this section is based on real hardware as shown in Fig. 5. The electric propulsion unit of the tri-rotor UAV includes energy storage units (battery packs), electronic speed control units (ESC), electric motors (brushless DC motors), and propellers. Also, an embedded system is installed on the main body, which includes an on-board microcontroller (OBM), a data acquisition module (DAQ) and a sensor module (IMU). The measured model parameters of the tri-rotor UAV are given in Table 1.

The simulation environment has been designed and implemented in Simscape Multibody ${ }^{\mathrm{TM}}$ and Simulink ${ }^{\circledR}$ for more realistic results as this provides a 3D graphical display of physical devices. Simscape Multibody ${ }^{\mathrm{TM}}$ is used to develop the dynamic model of the tri-rotor UAV based on physical components such as joints, constraints, force elements, and sensors. The designed control system is implemented in Simulink ${ }^{\circledR}$. Furthermore, a time delay of $0.01 \mathrm{~s}$ in servo motor responses and a maximum speed saturation constraint of 12000 RPM on the electric motors are considered in the simulation model to mimic real physical considerations. 
Table 1

Tri-rotor UAV and controller parameters

\begin{tabular}{|c|c|c|c|}
\hline & Value & Unit & Description \\
\hline $\mathrm{m}$ & 0.5 & $\mathrm{~kg}$ & Tri-rotor mass \\
\hline $\mathrm{g}$ & 9.81 & $\mathrm{~m} / \mathrm{s}^{2}$ & Gravity acceleration \\
\hline 1 & 0.23 & $\mathrm{~m}$ & Arm length \\
\hline$I_{x x}$ & $6.8 \times 10^{-3}$ & $\mathrm{~kg} \cdot \mathrm{m}^{2}$ & Moment of inertia along $X_{b}$ \\
\hline$I_{y y}$ & $5.3 \times 10^{-3}$ & $\mathrm{~kg} \cdot \mathrm{m}^{2}$ & Moment of inertia along $Y_{b}$ \\
\hline$I_{z z}$ & $1.7 \times 10^{-3}$ & $\mathrm{~kg} \cdot \mathrm{m}^{2}$ & Moment of inertia along $Z_{b}$ \\
\hline$I_{y z}$ & $3.1 \times 10^{-4}$ & $\mathrm{~kg} \cdot \mathrm{m}^{2}$ & Product of inertia about $Y_{b}$ and $Z_{b}$ \\
\hline$k_{f}$ & $1.97 \times 10^{-5}$ & $\mathrm{~kg} \cdot \mathrm{m} / \mathrm{rad}^{2}$ & Thrust to speed coefficient \\
\hline$k_{d}$ & $2.88 \times 10^{-7}$ & $\mathrm{~kg} \cdot \mathrm{m}^{2} / \mathrm{rad}^{2}$ & Drag to speed coefficient \\
\hline
\end{tabular}

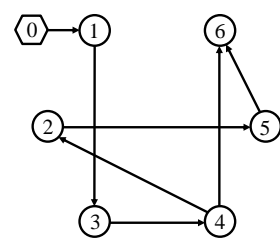

(a)

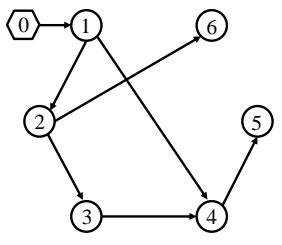

(b)

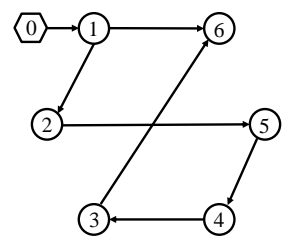

(c)
Fig. 6. Directed interaction topologies

For this case study, consider a set of six tri-rotor UAVs performing a target surveillance task, whose goal is to track a predefined time-varying formation while maintaining different attitudes individually for monitoring the full range of target activity. The directed interaction topology among the six vehicles is shown in Fig. 6, where the leader agent 0 provides the formation reference signal and the directed topology is switched every 5 seconds in sequence. Recall that $\boldsymbol{h}_{i} \in \mathbb{R}^{n}$ is the formation offset vector with respect to the formation reference $\boldsymbol{x}_{0} \in \mathbb{R}^{n}$. The 3D attitude and $3 \mathrm{D}$ position of each UAV are chosen independently.

The matrix $\overline{\boldsymbol{B}}$ can be chosen as

$$
\overline{\boldsymbol{B}}=\left[\begin{array}{llllllllllll}
0 & 0 & 0 & 0 & 0 & 0 & 1 & 0 & 0 & 0 & 0 & 0 \\
0 & 0 & 0 & 0 & 0 & 0 & 0 & 1 & 0 & 0 & 0 & 0 \\
0 & 0 & 0 & 0 & 0 & 0 & 0 & 0 & 1 & 0 & 0 & 0 \\
0 & 0 & 0 & 0 & 0 & 0 & 0 & 0 & 0 & 1 & 0 & 0 \\
0 & 0 & 0 & 0 & 0 & 0 & 0 & 0 & 0 & 0 & 1 & 0 \\
0 & 0 & 0 & 0 & 0 & 0 & 0 & 0 & 0 & 0 & 0 & 1
\end{array}\right],
$$

and $\tilde{\boldsymbol{B}}$ can be calculated as a left generalized inverse of $\boldsymbol{B}_{r}$, which is given by

$$
\tilde{\boldsymbol{B}}=\left[\begin{array}{cccccccccccc}
0 & 406.09 & 29.68 & 0 & -0.05 & 0.28 & 0 & 0 & 0 & 0 & 0 & 0 \\
-351.68 & -203.04 & 29.68 & 0 & -0.05 & 0.28 & 0 & 0 & 0 & 0 & 0 & 0 \\
351.68 & -203.04 & 29.68 & 0 & -0.05 & 0.28 & 0 & 0 & 0 & 0 & 0 & 0 \\
0 & -59.36 & 203.04 & 0 & -1.79 & 0.10 & 0 & 0 & 0 & 0 & 0 & 0 \\
51.41 & 29.68 & 203.04 & 1.99 & 0.89 & -0.05 & 0 & 0 & 0 & 0 & 0 & 0 \\
-51.41 & 29.68 & 203.04 & -1.99 & 0.89 & -0.05 & 0 & 0 & 0 & 0 & 0 & 0
\end{array}\right] .
$$

In this case, the states of the leader node are given by

$$
\boldsymbol{x}_{0}=\left[\begin{array}{lllllllllllll}
0 & 0 & 0 & 0 & 0 & 0 & 0 & 0 & 0 & 5 & -5 & 0
\end{array}\right]^{T},
$$

which indicates that the reference position will be located at a static position $(5,-5,0)$ with roll, pitch and yaw angles being zero, and all the reference velocities and reference angular velocities are kept zero to maintain the static target position. It should however be pointed out that the individual attitudes and positions of each UAV are also effected by the choice of $h_{i}$, which will not be set to zero. The proposed control strategy is valid regardless of the target is static or time-varying. In this

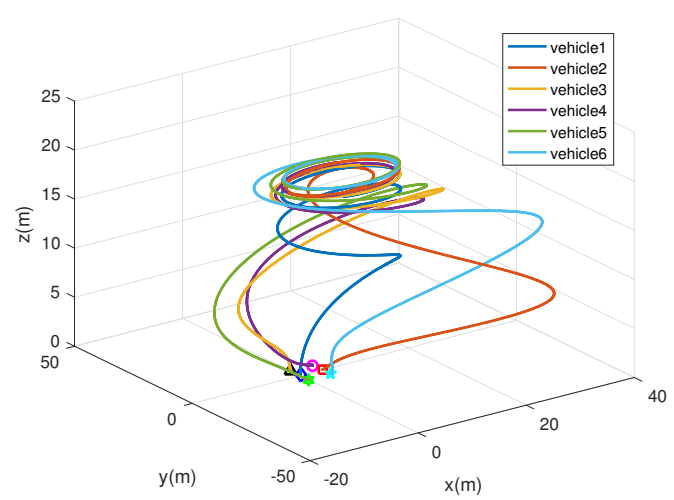

Fig. 7. 3D trajectories of the tri-rotor swarm

case, $\boldsymbol{h}_{i}$ is selected as

$$
\left.\boldsymbol{h}_{i}=\left[\begin{array}{c}
10 \cos \left(t+\frac{(i-1) \pi}{3}\right) \\
-10 \sin \left(t+\frac{(i-1) \pi}{3}\right) \\
0 \\
0 \\
0 \\
0 \\
-0.5 \times(-1)^{i} \\
-0.5 \times(-1)^{i} \\
0.5 \times(-1)^{i} \\
10 \sin \left(t+\frac{(i-1) \pi}{3}\right) \\
10 \cos \left(t+\frac{(i-1) \pi}{3}\right) \\
20
\end{array}\right] \text { (for } i \in\{1,2, \ldots, 6\}\right)
$$

where the desired offsets of 3D attitude and 3D position with respect to the reference signal for each agent are represented by last six rows, and the first six rows are the derivatives of them according to the change of coordinates given in (67).

It can be verified that the formation tracking feasibility condition (76) in Theorem 1 is satisfied. Then the optimal statefeedback gain $\boldsymbol{K}$ and coupling gain $c$ can be obtained using the approach in Theorem 2. The local optimal observer gain $\boldsymbol{F}$ for each UAV can also be selected easily by solving the corresponding ARE based on the estimation of noise. These design gains are hence given by

$$
c=5
$$

$$
\boldsymbol{K}=\left[\begin{array}{cccccccccccc}
-2.1 & 0.3 & 12 & -0.1 & -2.3 & 3.2 & -0.9 & -9.4 & 18 & -2.2 & 0.5 & 2.6 \\
2.1 & -2.9 & 12 & 2.6 & 1.2 & 3.1 & 11 & 7.6 & 17 & 2.8 & -3.5 & 2.6 \\
2.6 & 2.5 & 12 & -2.4 & 1.3 & 3.1 & -11 & 8.9 & 17 & 3.7 & 2.9 & 2.6 \\
-20 & 0 & 85 & 0 & -17 & 0.3 & 0.1 & -81 & -0.5 & -25 & 0 & 18 \\
10 & -18 & 85 & 17 & 8.4 & -0.8 & 77 & 40 & -3.6 & 12 & -22 & 18 \\
10 & 18 & 85 & -17 & 8.4 & -0.8 & -77 & 40 & -3.6 & 12 & 22 & 18
\end{array}\right]
$$

$$
\boldsymbol{F}=\left[\begin{array}{cccccc}
-0.12 & 10.08 & -0.01 & 9.83 & 0 & 0 \\
-10.14 & -0.12 & 0 & 0 & 9.83 & 0 \\
0 & 0 & -0.18 & 0 & 0 & 1.25 \\
160.26 & 0 & 0 & -0.12 & 5.57 & 0 \\
0 & 208.61 & 50.72 & -6.01 & -0.12 & 0 \\
0 & 50.72 & 916.32 & -0.13 & 0 & -0.18 \\
17.90 & 0 & 0 & -0.01 & -0.20 & 0 \\
0 & 20.36 & 1.60 & 0.16 & -0.01 & 0 \\
0 & 1.60 & 42.77 & 0 & 0 & 0 \\
-0.01 & 0.16 & 0 & 4.43 & 0 & 0 \\
-0.20 & -0.01 & 0 & 0 & 4.43 & 0 \\
0 & 0 & 0 & 0 & 0 & 1.5864
\end{array}\right] .
$$

On using robust control law (49) with optimal distributed formation control protocol (72) and (73), the trajectory of each tri-rotor UAV is given by Fig. 7. The 3D visualisation of distributed formation of the tri-rotor UAV swarm are illustrated in Fig. 8. The attitude tracking performance with respect to roll, 


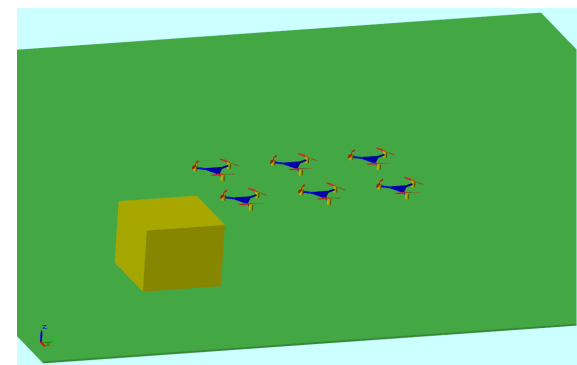

(a)

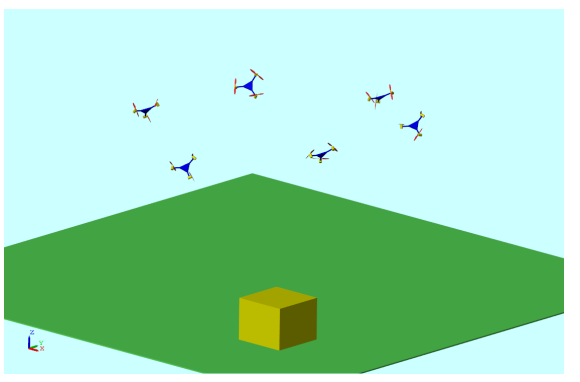

(d)

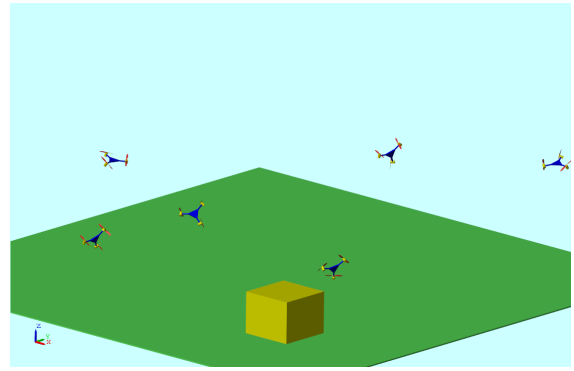

(b)

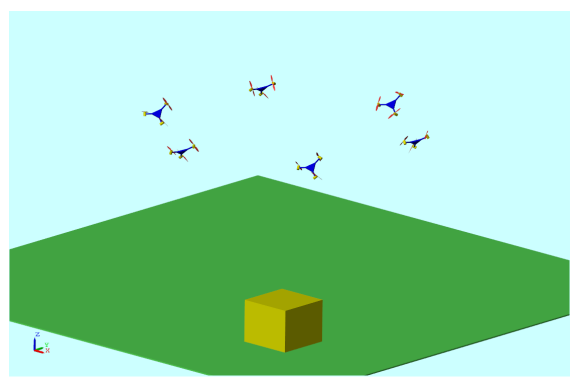

(e)

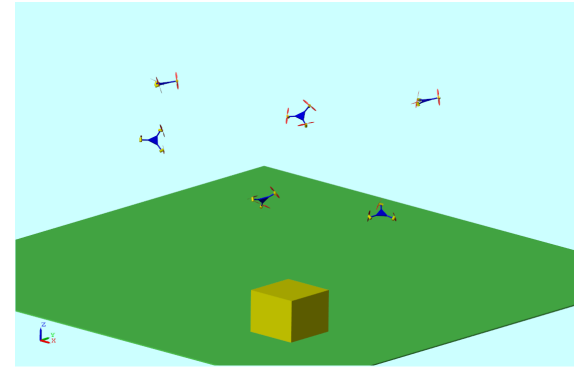

(c)

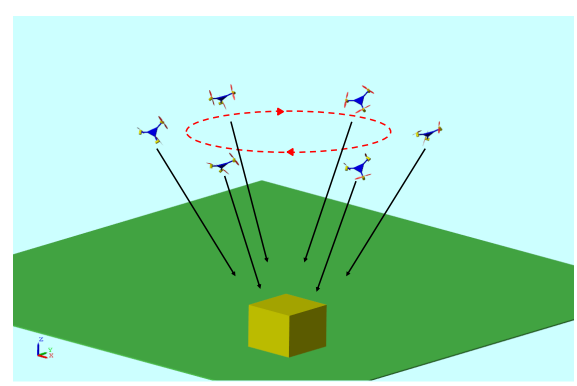

(f)

Fig. 8. $3 \mathrm{D}$ shots of the tri-rotor swarm. (a) $t=0 \mathrm{~s}$. (b) $t=5 \mathrm{~s}$. (c) $t=10 \mathrm{~s}$. (d) $t=15 \mathrm{~s}$. (e) $t=25 \mathrm{~s}$. (f) $t=50 \mathrm{~s}$.

pitch and yaw angles is shown in Fig. 9. The position tracking of hovering is shown in Fig. 10. From all these figures, it can be seen that the tri-rotor swarm forms a regular hexagonal formation with circular time-variation in the $x-y$ plane after $25 \mathrm{~s}$ and the surveilled target lies in the middle of the circular rotation at ground level. The attitude of each tri-rotor UAV varies with time along the circular trajectory so that each tri-rotor points (e.g. its onboard camera) to the target located at the centre of the circle. It is concluded that the desired formation and attitude tracking of the UAV swarm is achieved independently, and the designed control system preserves good robustness properties when subjected to simulated aerodynamic disturbances and model uncertainties.

\section{Conclusion}

In this paper, we have proposed a new tri-rotor unmanned aerial vehicle which is more efficient and flexible than a quadrotor UAV. A formation tracking problem of a networked tri-rotor UAV swarm has also been solved using a distributed formation control protocol.

To achieve this, the dynamical model was first derived based on force and torque kinematic analysis and subsequent translational and rotational dynamic modelling. A robust feedback linearization controller was then developed to deal with this highly coupled and nonlinear tri-rotor UAV to achieve a feedback linearized system through geometric transformation that is valid at any operating point but matches the Jacobi linearization of the system at the operating point of interest. The technique preserves robustness as it does not invert all nonlinear dynamics, unlike classic feedback linearization. An distributed optimal formation tracking control protocol was then developed for the tri-rotor robotic swarm, which guarantees that the target timevarying position and time-varying attitude of each UAV can be achieved independently. Finally, simulation results were given in a realistic environment based on 3D graphical display and physical visualisations. It has been shown that the proposed tri-rotor UAV swarm is able to track a desired time-varying formation whilst independently tracking different time-varying attitudes. A target surveillance task was performed effectively by these tri-rotor UAVs, which lays the foundation for some more complex collaborative tasks to be explored.

Future work will take obstacle avoidance and power management as shown in [43] into consideration, the proposed distributed controller will be applied to real hardware, and robust methods such as [44], [45] will be exploited in the design of the distributed control protocol.

\section{Acknowledgment}

This work was supported by the Engineering and Physical Sciences Research Council (EPSRC) [grant number EP/R008876/1]. All research data supporting this publication are directly available within this publication.

\section{References}

[1] R. Mahony, V. Kumar, P. Corke, Multirotor aerial vehicles: Modeling, estimation, and control of quadrotor, IEEE Robotics \& Automation Magazine 19 (3) (2012) 20-32.

[2] M. Senanayake, I. Senthooran, J. C. Barca, H. Chung, J. Kamruzzaman, M. Murshed, Search and tracking algorithms for swarms of robots: A survey, Robotics and Autonomous Systems 75 (2016) 422-434.

[3] P. Lottes, R. Khanna, J. Pfeifer, R. Siegwart, C. Stachniss, UAV-based crop and weed classification for smart farming, in: Proceeding of the 


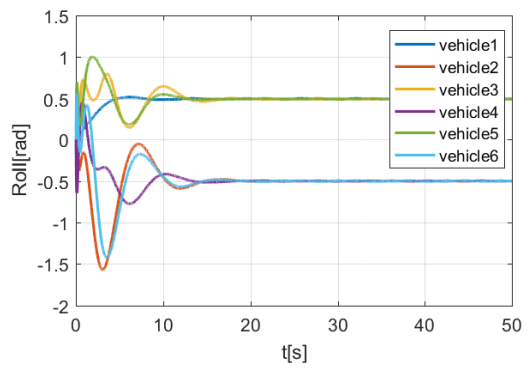

(a)

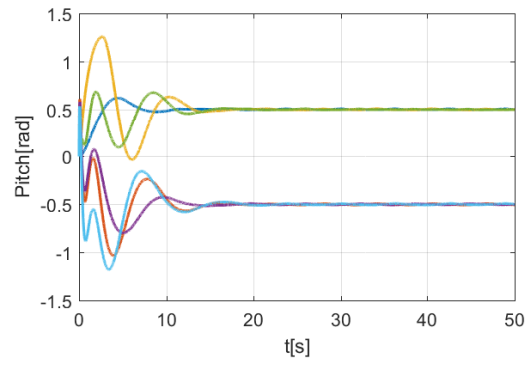

(b)

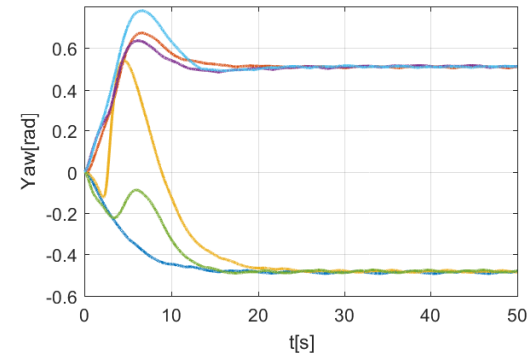

(c)

Fig. 9. Attitude response of the tri-rotor swarm system. (a) Roll angle $\phi$. (b) Pitch angle $\theta$. (c) Yaw angle $\psi$.

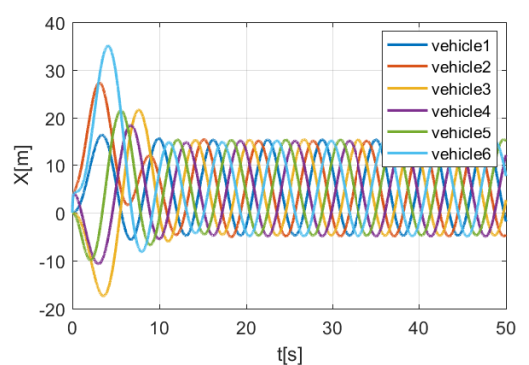

(a)

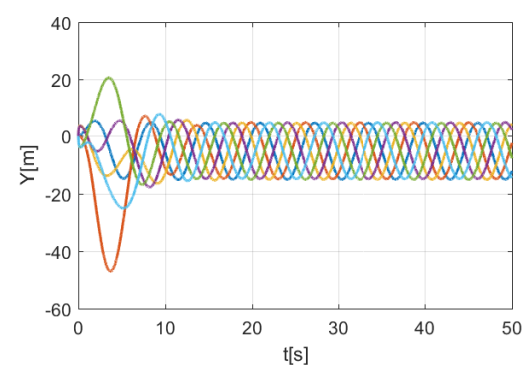

(b)

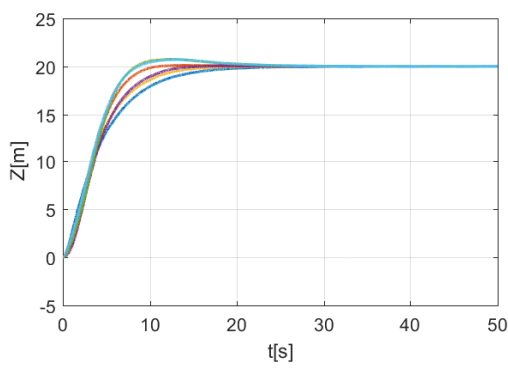

(c)

Fig. 10. Position response of the tri-rotor swarm system. (a) Longitudinal displacement $x_{v}$. (b) Lateral displacement $y_{v}$. (c) Vertical displacement $z_{v}$

IEEE International Conference on Robotics and Automation (ICRA), 2017, pp. 3024-3031.

[4] A. Shukla, H. Karki, Application of robotics in onshore oil and gas industrya review part I, Robotics and Autonomous Systems 75 (2016) 490-507.

[5] B. Crowther, A. Lanzon, M. Maya-Gonzalez, D. Langkamp, Kinematic analysis and control design for a nonplanar multirotor vehicle, AIAA Journal of Guidance, Control, and Dynamics 34 (4) (2011) 1157-1171.

[6] M. Kara Mohamed, A. Lanzon, Design and control of novel tri-rotor UAV, in: Proceedings of the 2012 UKACC International Conference on Control, Cardiff, UK, 2012, pp. 304-309.

[7] M. Kara Mohamed, A. Lanzon, Effect of unmodelled actuator dynamics on feedback linearised systems and a two stage feedback linearisation method, in: Proceedings of the IEEE Conference on Decision and Control (CDC), 2013, pp. 841-846.

[8] S. Driessens, P. Pounds, The triangular quadrotor: a more efficient quadrotor configuration, IEEE Transactions on Robotics 31 (6) (2015) 1517-1526.

[9] D.-W. Yoo, H.-D. Oh, D.-Y. Won, M.-J. Tahk, Dynamic modeling and stabilization techniques for tri-rotor unmanned aerial vehicles, International Journal Aeronautical and Space Sciences 11 (3) (2010) 167-174.

[10] J. Escareno, A. Sanchez, O. Garcia, R. Lozano, Triple tilting rotor miniUAV: Modeling and embedded control of the attitude, in: Proceedings of the American Control Conference, 2008, pp. 3476-3481.

[11] P. Rongier, E. Lavarec, F. Pierrot, Kinematic and dynamic modeling and control of a 3-rotor aircraft, in: Proceedings of the IEEE International Conference on Robotics and Automation (ICRA), 2005, pp. 2606-2611.

[12] I. Navarro, F. Matía, An introduction to swarm robotics, ISRN Robotics 2013.

[13] J. Wang, A. Lanzon, I. R. Petersen, Robust output feedback consensus for networked negative-imaginary systems, IEEE Transactions on Automatic Control 60 (9) (2015) 2547-2552.

[14] R. Aragues, J. Cortes, C. Sagues, Distributed consensus algorithms for merging feature-based maps with limited communication, Robotics and Autonomous Systems 59 (3) (2011) 163-180.

[15] J. Wang, M. Xin, Distributed optimal cooperative tracking control of multiple autonomous robots, Robotics and Autonomous systems 60 (4)
(2012) 572-583.

[16] R. Van Parys, G. Pipeleers, Distributed mpc for multi-vehicle systems moving in formation, Robotics and Autonomous Systems 97 (2017) 144 152.

[17] J. Wang, A. Lanzon, I. R. Petersen, Robust cooperative control of multiple heterogeneous negative-imaginary systems, Automatica 61 (2015) 64-72.

[18] M. H. Yamchi, R. M. Esfanjani, Distributed predictive formation control of networked mobile robots subject to communication delay, Robotics and Autonomous Systems 91 (2017) 194-207.

[19] W. Ren, N. Sorensen, Distributed coordination architecture for multirobot formation control, Robotics and Autonomous Systems 56 (4) (2008) 324-333.

[20] K. Ovchinnikov, A. Semakova, A. Matveev, Cooperative surveillance of unknown environmental boundaries by multiple nonholonomic robots, Robotics and Autonomous Systems 72 (2015) 164-180.

[21] M. Kubo, H. Sato, T. Yoshimura, A. Yamaguchi, T. Tanaka, Multiple targets enclosure by robotic swarm, Robotics and Autonomous Systems 62 (9) (2014) 1294-1304.

[22] H. Ebel, E. S. Ardakani, P. Eberhard, Distributed model predictive formation control with discretization-free path planning for transporting a load, Robotics and Autonomous Systems 96 (2017) 211-223.

[23] W. Ren, Consensus strategies for cooperative control of vehicle formations, IET Control Theory \& Applications 1 (2) (2007) 505-512.

[24] J. A. Fax, R. M. Murray, Information flow and cooperative control of vehicle formations, IEEE Transactions on Automatic Control 49 (9) (2004) 1465-1476.

[25] M. Turpin, N. Michael, V. Kumar, Decentralized formation control with variable shapes for aerial robots, in: Proceeding of the IEEE International Conference on Robotics and Automation (ICRA), 2012, pp. 23-30.

[26] A. L. D. Franco, H. Bourlès, E. R. De Pieri, H. Guillard, Robust nonlinear control associating robust feedback linearization and $H_{\infty}$ control, IEEE Transactions on Automatic Control 51 (7) (2006) 1200-1207.

[27] A. Lanzon, A. Freddi, S. Longhi, Flight control of a quadrotor vehicle subsequent to a rotor failure, AIAA Journal of Guidance, Control, and Dynamics 37 (2) (2014) 580-591.

[28] H. Zhang, F. L. Lewis, A. Das, Optimal design for synchronization of 
cooperative systems: state feedback, observer and output feedback, IEEE Transactions on Automatic Control 56 (8) (2011) 1948-1952.

[29] W. Ren, R. W. Beard, Consensus seeking in multiagent systems under dynamically changing interaction topologies, IEEE Transactions on Automatic Control 50 (5) (2005) 655-661.

[30] M. Bangura, R. Mahony, Thrust control for multirotor aerial vehicles, IEEE Transactions on Robotics 33 (2) (2017) 390-405.

[31] J. L. Meriam, L. G. Kraige, Engineering mechanics: dynamics, Vol. 2, John Wiley \& Sons, 2012.

[32] T. Fossen, Marine control systems: guidance, navigation and control of ships, rigs and underwater vehicles, Marine Cybernetics, 2002.

[33] A. Freddi, A. Lanzon, S. Longhi, A feedback linearization approach to fault tolerance in quadrotor vehicles, in: Proceedings of the 18th IFAC World Congress, Milan, Italy, 2011, pp. 5413-5418.

[34] A. Isidori, Nonlinear control systems, Springer-Verlag, 1995.

[35] X. Dong, G. Hu, Time-varying formation control for general linear multiagent systems with switching directed topologies, Automatica 73 (2016) 47-55.

[36] Z. Li, Z. Duan, G. Chen, L. Huang, Consensus of multiagent systems and synchronization of complex networks: A unified viewpoint, IEEE Transactions on Circuits and Systems I 57 (1) (2010) 213-224.

[37] C.-T. Chen, Linear system theory and design, Oxford University Press, Inc., 1995.

[38] D. Simon, Optimal state estimation: Kalman, $\mathrm{H}$ infinity, and nonlinear approaches, John Wiley \& Sons, 2006.

[39] R. E. Kalman, R. S. Bucy, New results in linear filtering and prediction theory, Journal of basic engineering 83 (3) (1961) 95-108.

[40] J. C. Doyle, K. Glover, P. P. Khargonekar, B. A. Francis, State-space solutions to standard $H_{2}$ and $H_{\infty}$ control problems, IEEE Transactions on Automatic Control 34 (8) (1989) 831-847.

[41] M. S. Grewal, Kalman filtering, in: International Encyclopedia of Statistical Science, Springer, 2011, pp. 705-708

[42] A. Dehghani, A. Lecchini-Visintini, A. Lanzon, B. D. Anderson, Validating controllers for internal stability utilizing closed-loop data, IEEE Transactions on Automatic Control 54 (11) (2009) 2719-2725.

[43] M. Patil, T. Abukhalil, S. Patel, T. Sobh, UB robot swarm - Design, implementation, and power management, in: proceedings of the IEEE International Conference on Control and Automation (ICCA), 2016, pp. $577-582$.
[44] A. Lanzon, Weight optimisation in $H_{\infty}$ loop-shaping, Automatica 41 (7) (2005) 1201-1208.

[45] A. Lanzon, G. Papageorgiou, Distance measures for uncertain linear systems: A general theory, IEEE Transactions on Automatic Control 54 (7) (2009) 1532-1547.

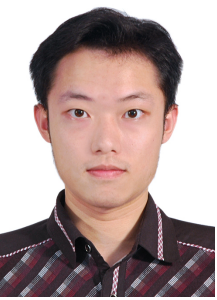

Junyan Hu received the B.Eng degree in Automation from Hefei University of Technology in 2015 and M.Sc degree in Advanced Control and System Engineering from the University of Manchester in 2016, where he is currently working toward the Ph.D degree in Electrical and Electronic Engineering. His research interests include distributed/formation control of multi-agent dynamical systems and control application for innovative aerial vehicles.

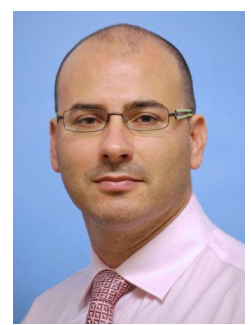

Alexander Lanzon received his Ph.D. degree in Control Engineering and his M.Phil. degree in Robot Control from the University of Cambridge in 2000 and 1997 respectively and received his B.Eng.(Hons). degree in Electrical and Electronic Engineering from the University of Malta in 1995. Alexander held academic positions at Georgia Institute of Technology and the Australian National University, and industrial posts at STMicroelectronics (Malta) Ltd., Yaskawa Denki (Tokyo) Ltd. and National ICT Australia Ltd., before joining the University of Manchester in 2006 where he now holds the Chair in Control Engineering. Alexander is a Fellow of the Institute of Mathematics and its Applications, the Institute of Measurement and Control and the Institution of Engineering and Technology. He is also an Associate Editor of the IEEE Transactions on Automatic Control and has served as a Subject Editor of the International Journal of Robust and Nonlinear Control. His research interests include negative imaginary systems, robust control, fundamentals of feedback control theory, distributed/consensus control of multi-agent dynamical systems, and control applications for innovative aerial drones, robotics and mechatronic systems. 